\title{
Supporting Information Reaction of Metalated Nitriles with Enones
}

\author{
Hans J. Reich, * Margaret Biddle and Robert Edmonston \\ Department of Chemistry, University of Wisconsin \\ Madison, Wisconsin 53706 \\ reich@chem.wisc.edu
}

Contents

S-2 Table S-1. Time and solvent dependence of $\mathbf{3}$ reacting with benzylideneacetone.

S-3 Table S-2. Effect of LiOPh on rearrangement of 9 to 10.

Table S-3. Time dependence of the rearrangement of $\mathbf{4}$ to 5 .

Characterization of $\mathbf{4}, \mathbf{5}$.

S-4 Table S-4. Diastereomer interconversion of $\mathbf{4 A}$ and $\mathbf{4 B}$.

S-5 Table S-5. Benzaldehyde trapping of $\mathbf{4}$ to form $\mathbf{1 1 .}$

Characterization of $\mathbf{1 1}$.

Figure S-1. First order rates of retro Michael, incorporation of 12 into 5.

S-6 Figure S-2. $k_{\mathrm{obs}}, \Delta G^{\ddagger}$, Eyring plot.

Characterization of keto-13.

Preparation and characterization of 17-H, 17-CN.

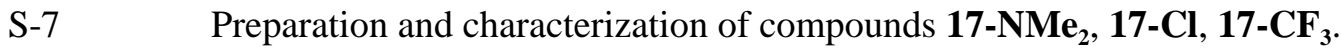

S-8 Table S-6. Time dependence of $\mathbf{1 7 - X}$ reacting with mesityl oxide.

S-9 Characterization of 20-X and 21-X.

S-10 Table S-7.Time dependence of lithio- 4-methoxyphenylacetonitrile reacting with mesityl oxide. Characterization of.1,2 and 1,4 adducts of lithio- 4-methoxyphenylacetonitrile with mesityl oxide.

S-11-S-35 $\quad{ }^{1} \mathrm{H}$ and ${ }^{13} \mathrm{C}$ NMR Spectra compounds 4A, 4B, 5, $\boldsymbol{d}$-10, $\boldsymbol{d}$-14, 11, keto-13, 17-X, 20-X, 21-X and 1,2 and 1,4 adducts of lithio- 4-methoxyphenylacetonitrile with mesityl oxide. 


\section{Experimental Section}

Table S-1. Time and solvent dependence of reaction of 3 with benzylideneacetone at $-78{ }^{\circ} \mathrm{C}$ (Fig. 3).

\begin{tabular}{|c|c|c|c|c|c|c|}
\hline Solvent & Time / sec & $4: 5$ & $4 d r$ & $5 d r$ & $\% \mathrm{SM}^{\mathrm{a}}$ & $\%$ Recovery \\
\hline $\mathrm{THF}^{\mathrm{b}}$ & 3 & $95: 5$ & $67: 33$ & - & 17 & 99 \\
\hline THF & 15 & $94: 6$ & $60: 40$ & - & 9 & 98 \\
\hline $\mathrm{THF}$ & 120 & $83: 17$ & $60: 40$ & $83: 17$ & 11 & 79 \\
\hline $\mathrm{THF}$ & 630 & $63: 37$ & $57: 43$ & $85: 15$ & 9 & 116 \\
\hline THF & 2400 & $41: 59$ & $54: 46$ & $80: 20$ & 4 & 98 \\
\hline $\mathrm{THF}$ & 5420 & $26: 74$ & $48: 52$ & $87: 13$ & 1 & 95 \\
\hline 1:1 THF/Tol & 5 & $85: 15$ & & & - & 88 \\
\hline 1:1 THF/Tol & 30 & $83: 17$ & & & - & 92 \\
\hline 1:1 THF/Tol & 60 & $81: 19$ & & & - & 88 \\
\hline 1:1 THF/Tol & 300 & $71: 29$ & & & - & 79 \\
\hline 1:1 THF/Tol & 600 & 66:34 & & & - & 96 \\
\hline 1:1 THF/Tol & 1200 & $53: 47$ & & & - & 91 \\
\hline 3:7 THF/Tol & 2 & $\begin{array}{c}91: 9 \\
\cdots \cdots \cdots\end{array}$ & & & 18 & 90 \\
\hline 3:7 THF/Tol & 15 & $88: 12$ & & & 0 & 120 \\
\hline 3:7 THF/Tol & 120 & $84: 16$ & & & 7 & 107 \\
\hline 3:7 THF/Tol & 300 & $77: 23$ & & & 12 & 135 \\
\hline 3:7 THF/Tol & 900 & 69:31 & & & 17 & 125 \\
\hline 3:7 THF/Tol & 1800 & $62: 38$ & & & 7 & 118 \\
\hline 3:7 THF/Tol & 3600 & 48:52 & & & 0 & 113 \\
\hline 3:7 THF/Tol & 14700 & $37: 63$ & & & 0 & 109 \\
\hline
\end{tabular}

${ }^{a}$ Recovered benzylideneacetone. ${ }^{\mathrm{b}}$ Dilute experiment at $0.001 \mathrm{M}$ concentration instead of standard $0.083 \mathrm{M}$. 
Table S-2. Time dependence of reaction of $\mathbf{3}$ with benzylideneacetone with one eq. of $\mathrm{LiOPh}$ in $\mathrm{THF}$ at $-78{ }^{\circ} \mathrm{C}$ (Fig. 3).

\begin{tabular}{|c|c|c|c|}
\hline Time $/ \mathrm{sec}$ & $\mathbf{4 : 5}$ & \% Recovery \\
\hdashline 60 & $91: 9$ & 117 \\
\hdashline 360 & $93: 7$ & 91 \\
\hdashline 1200 & $93: 7$ & 109 \\
\hdashline 2700 & $83: 17$ & 100 \\
\hline 1800 & $87: 13$ & 90 \\
\hdashline 3600 & $78: 22$ & 95 \\
\hline 5400 & $68: 32$ & 128 \\
\hdashline
\end{tabular}

Table S-3. Time dependence of reaction of 4 (9) in THF at $-78{ }^{\circ} \mathrm{C}$ rearranging to 5 (10) (Fig. 3).

\begin{tabular}{|c|c|c|}
\hline Time/sec & $\mathbf{4 : 5}$ & \% SM \\
\hdashline 15 & $93: 7$ & 34 \\
\hdashline 40 & $88: 12$ & 33 \\
\hdashline 120 & $73: 27$ & 18 \\
\hline 300 & $83: 17$ & 18 \\
\hdashline 635 & $65: 35$ & 27 \\
\hdashline 1200 & $49: 51$ & 7 \\
\hdashline 2400 & $35: 65$ & 16 \\
\hdashline 5400 & $20: 80$ & 13 \\
\hdashline & & 13
\end{tabular}

${ }^{a}$ Recovered benzylideneacetone.

3-Hydroxy-2,5-diphenyl-3-methylpent-4-enenitrile (4): ${ }^{1} \mathrm{H} \mathrm{NMR}\left(300 \mathrm{MHz}, \mathrm{CDCl}_{3}\right):(62: 38$

diastereomer ratio after $15 \mathrm{sec}$, major diastereomer marked with *): $\delta: 7.4-7.2(\mathrm{~m}, 10 \mathrm{H}), 6.62(\mathrm{~d}, \mathrm{~J}=16.0 \mathrm{~Hz}$, $1 \mathrm{H}), 6.58(\mathrm{~d}, \mathrm{~J}=16.0 \mathrm{~Hz}, 1 \mathrm{H})^{*}, 6.28(\mathrm{~d}, \mathrm{~J}=16.0 \mathrm{~Hz}, 1 \mathrm{H})^{*}, 6.27(\mathrm{~d}, \mathrm{~J}=16.0 \mathrm{~Hz}, 1 \mathrm{H}), 3.95(\mathrm{~s}, 1 \mathrm{H})^{*}, 3.92(\mathrm{~s}$, 1H), $2.27(\mathrm{~s}, \mathrm{OH})^{*}, 2.25(\mathrm{~s}, \mathrm{OH}), 1.52(\mathrm{~s}, 3 \mathrm{H}), 1.49(\mathrm{~s}, 3 \mathrm{H}) * .{ }^{13} \mathrm{C} \mathrm{NMR}\left(75.4 \mathrm{MHz}, \mathrm{CDCl}_{3}\right): \delta: 135.9(\mathrm{C})$, $131.7(\mathrm{C}), 131.6(\mathrm{C}), 131.3(\mathrm{CH}), 130.4(\mathrm{CH}), 130.3(\mathrm{CH}), \delta 129.5(\mathrm{CH}), \delta 128.6(\mathrm{CH}), 128.0(\mathrm{CH}), 126.6$ $(\mathrm{CH}), 119.3(\mathrm{CN}), 119.2(\mathrm{CN}), 74.1(\mathrm{C}), 74.1(\mathrm{C}), 50.1(\mathrm{CH}), 50.0(\mathrm{CH}), 26.1\left(\mathrm{CH}_{3}\right), 25.8\left(\mathrm{CH}_{3}\right)$. IR (neat): $3605 \mathrm{~cm}-1$ (free $\mathrm{OH}), 2241 \mathrm{~cm}-1(\mathrm{CN}) .\left[\mathrm{M}^{+}\right]=263.1297$, (calc. for $\mathrm{C}_{18} \mathrm{H}_{17} \mathrm{NO}, 263.1310$ ).

5-Oxo-2,3-diphenylhexanenitrile (5): ${ }^{1} \mathrm{H} \mathrm{NMR}\left(300 \mathrm{MHz}, \mathrm{CDCl}_{3}\right),(83: 17$ diastereomer ratio after 90 min, major diastereomer marked with *): $\delta$ 7.4-6.9 $(\mathrm{m}, 10 \mathrm{H}), 4.34(\mathrm{~d}, \mathrm{~J}=6.0 \mathrm{~Hz}, 1 \mathrm{H}) *, 4.09(\mathrm{~d}, \mathrm{~J}=6.5 \mathrm{~Hz}$, $1 \mathrm{H}), 3.71(\mathrm{ddd}, \mathrm{J}=9.0,6.0,5.0 \mathrm{~Hz}, 1 \mathrm{H}), 3.61(\mathrm{dt}, \mathrm{J}=8.5,6.0 \mathrm{~Hz}, 1 \mathrm{H})^{*}, 3.17(\mathrm{dd}, \mathrm{J}=17.0,8.5 \mathrm{~Hz}, 1 \mathrm{H})^{*}, 3.14$ $(\mathrm{dd}, \mathrm{J}=17.0,9.0 \mathrm{~Hz}, 1 \mathrm{H}), 2.97(\mathrm{dd}, \mathrm{J}=17.0,6.0 \mathrm{~Hz}, 1 \mathrm{H})^{*}, 2.90(\mathrm{dd}, \mathrm{J}=17.0,5.0 \mathrm{~Hz}, 1 \mathrm{H}), 2.15(\mathrm{~s}, 3 \mathrm{H})^{*}, 2.05$ $(\mathrm{s}, 3 \mathrm{H}) .{ }^{13} \mathrm{C}$ NMR $\left(75.4 \mathrm{MHz}, \mathrm{CDCl}_{3}\right)$ : $\delta 206.1(\mathrm{C}), 205.4(\mathrm{C}), 139.2(\mathrm{C}), 138.0(\mathrm{C}), 133.8(\mathrm{C}), 133.4(\mathrm{C})$, $128.7(\mathrm{CH}), 128.5(\mathrm{CH}), 128.3(\mathrm{CH}), 128.2(\mathrm{CH}), 127.9(\mathrm{CH}), 127.8(\mathrm{CH}), 127.6(\mathrm{CH}), 119.4(\mathrm{CN}), 119.2$ $(\mathrm{CN}), 46.3\left(\mathrm{CH}_{2}\right), 45.2\left(\mathrm{CH}_{2}\right), 44.9(\mathrm{CH}), 44.8(\mathrm{CH}), 43.7(\mathrm{CH}), 42.8(\mathrm{CH}), 30.5\left(\mathrm{CH}_{3}\right)$. IR (neat): 2240 cm-1 $(\mathrm{CN}), 1720(\mathrm{C}=\mathrm{O})$. $\mathrm{M}+=263.1313$, (calc. for $\left.\mathrm{C}_{18} \mathrm{H}_{17} \mathrm{NO}, 263.1310\right)$. 
Table S-4. Diastereomer interconversion of $4 \mathrm{~A}$ or $4 \mathrm{~B}$ at $-78^{\circ} \mathrm{C}$ in THF (Fig. 4 , measurement of $k_{-1,2}$ ).

\begin{tabular}{|c|c|c|}
\hline Time / sec & $\% \mathbf{4 A}$ in $\mathrm{SM}$ & $\% \mathbf{4 A}$ after rxn \\
\hline 5 & 20 & 37 \\
\hline 10 & 20 & 46 \\
\hline 30 & 20 & 46 \\
\hline 45 & 20 & 48 \\
\hline 60 & 20 & 54 \\
\hline 90 & 20 & 56 \\
\hline 120 & 20 & 54 \\
\hline 5 & 93 & 83 \\
\hline 10 & 93 & 60 \\
\hline 30 & 93 & 74 \\
\hline 60 & 93 & 67 \\
\hline 90 & 93 & 68 \\
\hline
\end{tabular}


Table S-5. Time dependence of benzaldehyde trapping of $\mathbf{3}$ at $-78{ }^{\circ} \mathrm{C}$ in THF (measurement of $k_{-12}$ ).

\begin{tabular}{|c|c|c|c|c|}
\hline Time / sec & 4:11 & $\% 4$ & $\% 11$ (anti:syn) & $\%$ Benzylideneacetone \\
\hline 4 & $87: 13$ & 76 & $11(83: 17)$ & 23 \\
\hline 5 & $78: 22$ & 74 & $21(85: 15)$ & 24 \\
\hline 5 & $80: 20$ & 74 & $19(79: 21)$ & 20 \\
\hline 5 & $62: 38$ & 48 & $29(74: 26)$ & 31 \\
\hline 5 & $67: 33$ & 62 & $31(81: 19)$ & 32 \\
\hline 6 & $76: 24$ & 73 & $23(78: 22)$ & 25 \\
\hline 10 & $73: 27$ & 77 & $28(81: 19)$ & 29 \\
\hline 10 & $64: 36$ & 56 & $32(80: 20)$ & 36 \\
\hline 10 & 78:22 & 78 & $22(85: 15)$ & 21 \\
\hline 12 & $54: 46$ & 44 & $37(87: 13)$ & 44 \\
\hline 13 & $48: 52$ & 35 & $38(79: 21)$ & 30 \\
\hline 20 & $93: 7$ & 93 & $7(83: 17)$ & 7 \\
\hline 20 & $68: 32$ & 60 & $28(75: 25)$ & 27 \\
\hline 20 & $60: 40$ & 66 & $44(83: 17)$ & 43 \\
\hline 30 & $58: 42$ & 33 & $46(77: 23)$ & 44 \\
\hline 40 & $51: 49$ & 51 & $49(84: 16)$ & 47 \\
\hline 40 & $55: 45$ & 38 & $31(78: 22)$ & 33 \\
\hline 40 & 49:51 & 52 & $55(82: 18)$ & 55 \\
\hline 60 & 62:38 & 50 & $31(82: 18)$ & 31 \\
\hline 60 & 40:60 & 39 & $58(79: 21)$ & 55 \\
\hline 61 & 44:56 & 41 & $52(76: 24)$ & 50 \\
\hline 120 & $17: 83$ & 13 & $64(82: 18)$ & 65 \\
\hline
\end{tabular}

3-Hydroxy-2,3-diphenylpropionitrile (11). ${ }^{1} \mathrm{H} \mathrm{NMR}\left(300 \mathrm{MHz}, \mathrm{CDCl}_{3}\right),(79: 21$ anti:syn diastereomer ratio after $5 \mathrm{sec}$, anti diastereomer marked with *): $\delta$ 7.18-7.35 $(\mathrm{m}, 10 \mathrm{H}), 4.99(\mathrm{~d}, \mathrm{~J}=7 \mathrm{~Hz}, 1 \mathrm{H}), 4.96(\mathrm{~d}, \mathrm{~J}=$ $6 \mathrm{~Hz}, 1 \mathrm{H})^{*}, 4.15(\mathrm{~d}, \mathrm{~J}=7 \mathrm{~Hz}, 1 \mathrm{H}), 4.04(\mathrm{~d}, \mathrm{~J}=6 \mathrm{~Hz}, 1 \mathrm{H})^{*}, 3.22$ (bs, 1H). ${ }^{13} \mathrm{C}$ NMR $\left(75.4 \mathrm{MHz}, \mathrm{CDCl}_{3}\right): \delta$ 139.4*(C), $138.8(\mathrm{C}), 32.4 *(\mathrm{C}), 131.9(\mathrm{C}), 128.80 *(\mathrm{CH}), 128.76(\mathrm{CH}), 128.7(\mathrm{CH}), 128.6 *(\mathrm{CH}), 128.5$ $(\mathrm{CH}), 128.43^{*}(\mathrm{CH}), 128.41(\mathrm{CH}), 128.39 *(\mathrm{CH}), 128.37 *(\mathrm{CH}), 128.32(\mathrm{CH}), 126.6(\mathrm{CH}), 126.20 *(\mathrm{CH})$, $126.19(\mathrm{CH}), 118.8^{*}(\mathrm{CN}), 118.7(\mathrm{CN}), 76.2^{*}(\mathrm{CH}), 76.0(\mathrm{CH}), 47.3 *(\mathrm{CH}), 46.5(\mathrm{CH})$. HRMS (EI) $(\mathrm{m} / \mathrm{z})$ :calcd. for $\mathrm{C}_{15} \mathrm{H}_{11} \mathrm{~N}\left(\mathrm{M}-\mathrm{H}_{2} \mathrm{O}^{+}\right)$, 205.0891; found 205.0890 . 
Figure S-1. First order rate plots of incorporation of $m$-methoxyphenylacetonitrile (12) into 1,4 adduct (5) at various temperatures (Scheme 2, measurement of $k_{-1,4}$ ). ${ }^{[a]}$

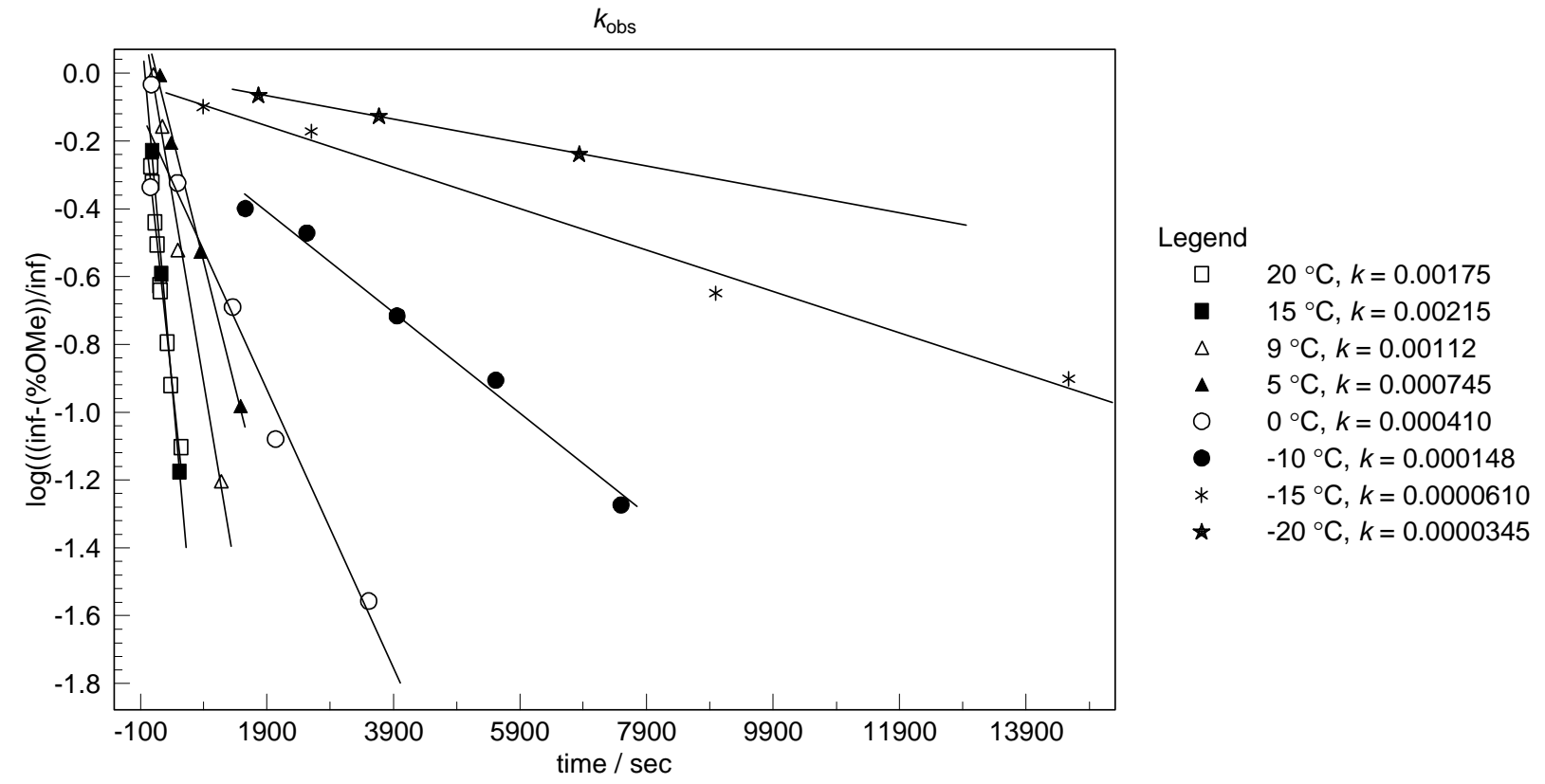

[a] Points shown were up to $70 \%$ conversion (100\% conversion corresponds to $66 \%$ incorporation of 12).

Figure S-2: Extrapolation of $\Delta G^{\ddagger}$ vs. T to give $\Delta G^{\ddagger}-78=18.7 \mathrm{kcal} / \mathrm{mol}, \Delta \mathrm{H}^{\ddagger}=15.0 \pm 1.0 \mathrm{kcal} / \mathrm{mol}$, and $\Delta S^{\ddagger}=19$ \pm 3.7 e.u.

\begin{tabular}{|c|c|c|}
\hline $\mathrm{T}$ in ${ }^{\circ} \mathrm{K}$ & $k_{\mathrm{obs}}$ in $\mathrm{sec}^{-1}$ & $\Delta G^{*}$ in $\mathrm{kcal} / \mathrm{mol}$ \\
\hline 293 & 0.00175 & 20.85 \\
\hline 288 & 0.00215 & 20.36 \\
\hline 282 & 0.00112 & 20.29 \\
\hline 278 & 0.000745 & 20.22 \\
\hline 273 & 0.000410 & 20.17 \\
\hline 263 & 0.000148 & 19.95 \\
\hline 258 & 0.0000610 & 20.01 \\
\hline 253 & 0.0000345 & 19.90 \\
\hline 195 & Extrapolated & 18.73 \\
\hline
\end{tabular}

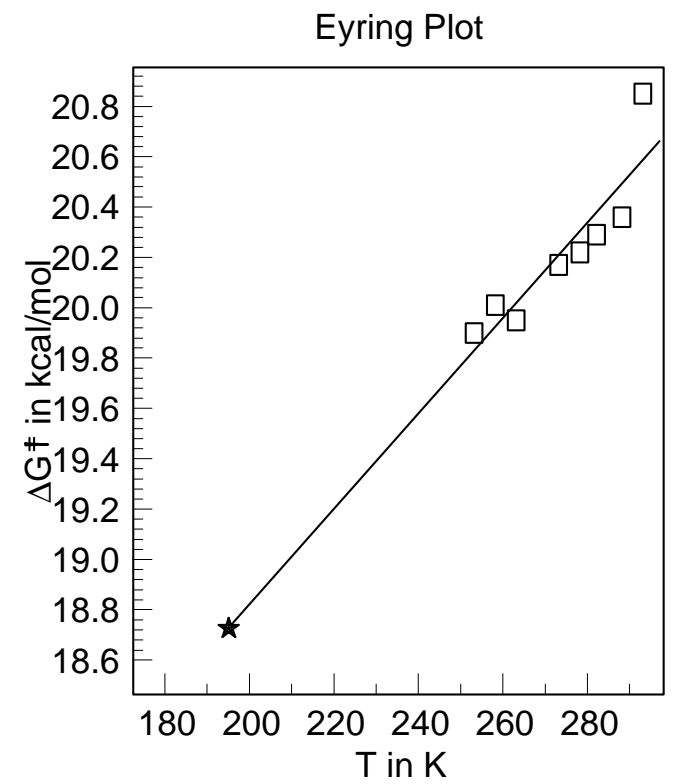

2-(3-Methoxyphenyl)-5-oxo-3-phenylhexanenitrile (keto-13). (73:27 dr after $85 \mathrm{sec}$ at $20{ }^{\circ} \mathrm{C}$, major diastereomer marked with *) ${ }^{1} \mathrm{H}$ NMR $\left(300 \mathrm{MHz}, \mathrm{CDCl}_{3}\right)$ : $\delta 7.4-6.9(\mathrm{~m}, 10 \mathrm{H}), 4.30(\mathrm{~d}, \mathrm{~J}=6.5 \mathrm{~Hz}, 1 \mathrm{H})^{*}, 4.06$ $(\mathrm{d}, \mathrm{J}=6.5 \mathrm{~Hz}, 1 \mathrm{H}), 3.71(\mathrm{ddd}, \mathrm{J}=\mathrm{Hz}, 1 \mathrm{H}), 3.61(\mathrm{dt}, \mathrm{J}=\mathrm{Hz}, 1 \mathrm{H})^{*}, 3.17(\mathrm{dd}, \mathrm{J}=17.0,8.5 \mathrm{~Hz}, 1 \mathrm{H})^{*}, 3.14(\mathrm{dd}, \mathrm{J}$ $=17.0,9.0 \mathrm{~Hz}, 1 \mathrm{H}), 2.97(\mathrm{dd}, \mathrm{J}=17.0,6.0 \mathrm{~Hz}, 1 \mathrm{H})^{*}, 2.90(\mathrm{dd}, \mathrm{J}=17.0,5.0 \mathrm{~Hz}, 1 \mathrm{H}), 2.15(\mathrm{~s}, 3 \mathrm{H})^{*}, 2.05(\mathrm{~s}$, 3H). ${ }^{13} \mathrm{C}$ NMR (75.4 MHz, $\left.\mathrm{CDCl}_{3}\right): \delta 206.1 *(\mathrm{C}), 205.5$ (C), 159.6*(C), $139.3(\mathrm{C}), 138.1 *(\mathrm{C}), 135.3 *(\mathrm{C})$, 
$134.9(\mathrm{C}), 129.8(\mathrm{CH}), 129.7 *(\mathrm{CH}), 128.7(\mathrm{CH}), 128.4 *(\mathrm{CH}), 128.3,127.9(\mathrm{CH}), 127.7 *(\mathrm{CH}), 120.34 *(\mathrm{CH})$, 120.30(CH), $119.4(\mathrm{CN}), 119.2^{*}(\mathrm{CN}), 114.1^{*}(\mathrm{CH}), 113.9(\mathrm{CH}), 113.8(\mathrm{CH}), 113.3(\mathrm{CH}), 55.2^{*}\left(\mathrm{CH}_{3}\right), 46.4$ $\left(\mathrm{CH}_{2}\right), 45.2\left(\mathrm{CH}_{2}\right), 45.0^{*}(\mathrm{CH}), 44.9(\mathrm{CH}), 43.9(\mathrm{CH}), 42.9^{*}(\mathrm{CH}), 30.6^{*}\left(\mathrm{CH}_{3}\right)$. HRMS (EI) (m/z): calcd. for $\mathrm{C}_{19} \mathrm{H}_{19} \mathrm{NO}_{2}\left(\mathrm{M}^{+}.\right), 293.1416$; found 293.1419.

Preparation of 4-Substituted phenylmethoxyacetonitriles (17-X): Sample Procedure. Phenylmethoxyacetonitrile (17-H). Prepared according to a standard procedure for making methyl ethers of cyanohydrins ${ }^{[28]}$ from $0.761 \mathrm{~g}(5.0 \mathrm{mmol})$ of the dimethyl acetal of benzaldehyde, ${ }^{[30]} 668 \mu \mathrm{L}(5.0 \mathrm{mmol})$ of trimethylsilyl cyanide, and a catalytic amount $(66 \mathrm{mg}, 0.34 \mathrm{mmol})$ of anhydrous $\mathrm{SnCl}_{2}$, allowed to stir $3 \mathrm{~h}$ at 20 ${ }^{\circ} \mathrm{C}$, taken up in $20 \mathrm{~mL}$ of diethyl ether, washed with once with $20 \mathrm{~mL}$ of saturated sodium bicarbonate solution, twice with $20 \mathrm{~mL}$ of water, once with $20 \mathrm{~mL}$ of brine and dried with anhydrous magnesium sulfate and rotary evaporated and run on a silica gel column eluted with 3:4 hexanes:diethyl ether to give $0.283 \mathrm{~g}$ (38\% yield). ${ }^{1} \mathrm{H}$ NMR $\left(300 \mathrm{MHz}, \mathrm{CDCl}_{3}\right): \delta 7.43-7.41(\mathrm{~m}, 5 \mathrm{H}), 5.19(\mathrm{~s}, 1 \mathrm{H}), 3.53(\mathrm{~s}, 3 \mathrm{H}) .{ }^{13} \mathrm{C}(75.44 \mathrm{MHz}$, $\left.\mathrm{CDCl}_{3}\right): \delta 133.3(\mathrm{C}), 129.8(\mathrm{CH}), 129.0(\mathrm{CH}), 127.2(\mathrm{CH}), 116.9(\mathrm{C}), 72.3\left(\mathrm{CH}_{2}\right), 57.1\left(\mathrm{CH}_{3}\right) . \mathrm{HRMS}(\mathrm{EI})$ $(\mathrm{m} / \mathrm{z})$ : calcd. for $\mathrm{C}_{9} \mathrm{H}_{9} \mathrm{NO}\left(\mathrm{M}^{+}\right), 147.0684$; found 147.0689.

(4-Cyanophenyl)methoxyacetonitrile (17-CN). Prepared according to a standard procedure for making methyl ethers of cyanohydrins ${ }^{[28]}$ from $0.605 \mathrm{~g}(3.33 \mathrm{mmol})$ of the dimethyl acetal of 4 -cyanobenzaldehyde, ${ }^{[30]}$ $445 \mu \mathrm{L}(3.33 \mathrm{mmol})$ of trimethylsilyl cyanide, and a catalytic amount $(44.2 \mathrm{mg}, 0.23 \mathrm{mmol})$ of anhydrous $\mathrm{SnCl}_{2}$, stirred $3 \mathrm{~h}$, to give $0.478 \mathrm{~g}\left(83.4 \%\right.$ yield). ${ }^{1} \mathrm{H} \mathrm{NMR}\left(300 \mathrm{MHz}, \mathrm{CDCl}_{3}\right): \delta 7.76-7.62\left(\mathrm{AA}^{\prime} \mathrm{BB}, 4 \mathrm{H}\right)$, $5.26(\mathrm{~s}, 1 \mathrm{H}), 3.60(\mathrm{~s}, 3 \mathrm{H}) .{ }^{13} \mathrm{C}\left(75.44 \mathrm{MHz}, \mathrm{CDCl}_{3}\right): \delta 138.1(\mathrm{C}), 132.8(\mathrm{CH}), 127.7(\mathrm{CH}), 117.9(\mathrm{C}), 116.0$ (C), $113.8(\mathrm{C}), 71.4\left(\mathrm{CH}_{2}\right), 57.8\left(\mathrm{CH}_{3}\right)$. HRMS (EI) (m/z): calcd.for $\mathrm{C}_{10} \mathrm{H}_{8} \mathrm{~N}_{2} \mathrm{O}\left(\mathrm{M}^{+}\right), 172.0637$; found 172.0636 .

(4-Dimethylamino-phenyl)methoxyacetonitrile (17-NMe $)$. Prepared according to a standard procedure for making methyl ethers of cyanohydrins ${ }^{[28]}$ from $1.405 \mathrm{~g}(7.2 \mathrm{mmol})$ of the dimethyl acetal of 4dimethylaminobenzaldehyde, ${ }^{[30]} 962 \mu \mathrm{L}(7.2 \mathrm{mmol})$ of trimethylsilyl cyanide, and a catalytic amount (96 mg, $0.50 \mathrm{mmol})$ of anhydrous $\mathrm{SnCl}_{2}$, stirred $12 \mathrm{~h}$, to give $0.650 \mathrm{~g}(47.4 \%$ yield $) .{ }^{1} \mathrm{H} \mathrm{NMR}\left(300 \mathrm{MHz}, \mathrm{CDCl}_{3}\right): \delta$ 7.34- 7.31, 6.73-6.71 (AA'BB', 4H), $5.10(\mathrm{~s}, 1 \mathrm{H}), 3.47(\mathrm{~s}, 3 \mathrm{H}), 2.98(\mathrm{~s}, 6 \mathrm{H}) .{ }^{13} \mathrm{C}\left(75.44 \mathrm{MHz}, \mathrm{CDCl}_{3}\right): \delta$ $151.3(\mathrm{C}), 128.6(\mathrm{CH}), 120.2(\mathrm{C}), 117.4(\mathrm{CN}), 112.0(\mathrm{CH}), 72.0(\mathrm{CH}), 56.4\left(\mathrm{CH}_{3}\right), 40.2\left(\mathrm{CH}_{3}\right) . \mathrm{HRMS}(\mathrm{ESI})$ $(\mathrm{m} / \mathrm{z})$ : calcd. for $\mathrm{C}_{11} \mathrm{H}_{15} \mathrm{ON}_{2}\left(\mathrm{MH}^{+}\right)$, 191.1184; found 191.1184.

(4-Chloro-phenyl)methoxyacetonitrile (17-Cl). Prepared according to a standard procedure for making methyl ethers of cyanohydrins ${ }^{[28]}$ from $0.267 \mathrm{~g}(1.43 \mathrm{mmol})$ of the dimethyl acetal of 4-chlorobenzaldehyde, ${ }^{[30]}$ $190 \mu \mathrm{L}(1.43 \mathrm{mmol})$ of trimethylsilyl cyanide, and a catalytic amount (19 mg, $0.10 \mathrm{mmol})$ of anhydrous $\mathrm{SnCl}_{2}$, stirred $5 \mathrm{~h}$, to give $0.202 \mathrm{~g}\left(78.2 \%\right.$ yield). ${ }^{1} \mathrm{H} \mathrm{NMR}\left(300 \mathrm{MHz}, \mathrm{CDCl}_{3}\right): \delta 7.43\left(\mathrm{AA}^{\prime} \mathrm{BB} ', 4 \mathrm{H}\right), 5.18(\mathrm{~s}, 1 \mathrm{H})$, $3.55(\mathrm{~s}, 3 \mathrm{H}) .{ }^{13} \mathrm{C}\left(\mathrm{NMR}\left(75.4 \mathrm{MHz}, \mathrm{CDCl}_{3}\right): \delta 135.9(\mathrm{C}), 131.8(\mathrm{C}), 129.2(\mathrm{CH}), 128.6(\mathrm{CH}), 116.6(\mathrm{CN})\right.$, $71.5(\mathrm{CH}), 57.3\left(\mathrm{CH}_{3}\right)$. HRMS (EI) $(\mathrm{m} / \mathrm{z})$ : calcd. for $\mathrm{C}_{8} \mathrm{H}_{5} \mathrm{CIN}\left(\mathrm{M}-\mathrm{OMe}^{+}\right), 150.0111$; found 150.0115.

Methoxy-(4-trifluoromethylphenyl)acetonitrile $\left(\mathbf{1 7 -}-\mathbf{C F}_{3}\right)$. Prepared according to a standard procedure for making methyl ethers of cyanohydrins, ${ }^{[28]}$ using $1.865 \mathrm{~g}(8.47 \mathrm{mmol})$ of the dimethyl acetal of 4trifluoromethylbenzaldehyde, ${ }^{[30]} 1.13 \mathrm{~mL}(8.47 \mathrm{mmol})$ of trimethylsilyl cyanide, and a catalytic amount $(112$ $\mathrm{mg}, 0.59 \mathrm{mmol})$ of anhydrous $\mathrm{SnCl}_{2}$, stirred $3 \mathrm{~h}$, to give $0.704 \mathrm{~g}(38.6 \%$ yield $)$. ${ }^{1} \mathrm{H} \mathrm{NMR}\left(300 \mathrm{MHz}, \mathrm{CDCl}_{3}\right): \delta$ 7.62-7.73 (AA'BB', 4H), $5.26(\mathrm{~s}, 1 \mathrm{H}), 3.59(\mathrm{~s}, 3 \mathrm{H}) .{ }^{13} \mathrm{C}\left(\mathrm{NMR}\left(90.6 \mathrm{MHz}, \mathrm{CDCl}_{3}\right): \delta 137.0(\mathrm{C}), 131.8 \mathrm{C},{ }^{2} \mathrm{~J}_{\mathrm{C}-}\right.$ $\mathrm{F}=32.5 \mathrm{~Hz}), 127.5(\mathrm{CH}), 126.0\left({ }^{3} \mathrm{CF}_{3} \mathrm{~J}_{\mathrm{C}-\mathrm{F}}=4 \mathrm{~Hz}\right), 123.6\left(\mathrm{CF}_{3}{ }^{1} \mathrm{~J}_{\mathrm{C}-\mathrm{F}}=273 \mathrm{~Hz}\right) 116.3(\mathrm{CN}), 71.53(\mathrm{CH}), 57.6$ $\left(\mathrm{CH}_{3}\right)$. HRMS(EI) (m/z): calcd. for $\mathrm{C}_{10} \mathrm{H}_{8} \mathrm{~F}_{3} \mathrm{ON}\left(\mathrm{M}^{+}\right)$, 215.0558; found 215.0567. 
Table S-6. Time dependence of reaction of 17-X with mesityl oxide (Fig. 6).

\begin{tabular}{|c|c|c|c|c|c|c|c|c|}
\hline Nucleophile & 20-X:21-X & Time / sec & $\% 1,2$ & $\% 1,4$ & $\% \mathrm{RXN}$ & \%recov. & $\mathrm{T}$ in ${ }^{\circ} \mathrm{C}$ & Solvent \\
\hline $17-\mathrm{CF}_{3}$ & $50: 50$ & 6 & 5 & 5 & 12 & 86 & -78 & THF \\
\hline 17- $\mathbf{C F}_{3}$ & $24: 74$ & 8 & 11 & 34 & 43 & 105 & -78 & THF \\
\hline $17-\mathbf{C F}_{3}$ & $12: 88$ & 25 & 5 & 36 & 46 & 90 & -78 & THF \\
\hline $17-\mathrm{CF}_{3}$ & 7:93 & 60 & 6 & 82 & 81 & 108 & -78 & THF \\
\hline 17-CF & $85: 15$ & 10 & 17 & 3 & 20 & 101 & -120 & 3:2 THF:Et2O \\
\hline 17-CI & $96: 4$ & 5 & 48 & 2 & 60 & 83 & -78 & THF \\
\hline 17-CI & $75: 25$ & 30 & 46 & 15 & 73 & 83 & -78 & THF \\
\hline 17-Cl & 40:60 & 120 & 25 & 37 & 77 & 81 & -78 & THF \\
\hline 17-Cl & $18: 82$ & 900 & 12 & 55 & 85 & 79 & -78 & THF \\
\hline 17-Cl & $100: 0$ & 5 & 43 & 0 & 40 & 107 & -78 & DME \\
\hline 17-CN & $0: 100$ & 40 & 7 & 26 & 35 & 93.5 & -78 & THF \\
\hline 17-CN & $0: 100$ & 360 & 0 & 87.2 & 84 & 104 & -78 & THF \\
\hline 17-CN & $0: 100$ & 1860 & 0 & 98 & 98 & 100 & -78 & THF \\
\hline 17-NMe & 100:0 & 30 & 100 & 0 & 100 & 100 & -78 & THF \\
\hline 17-NMe & 100:0 & 600 & 89 & 0 & 100 & 89 & -78 & THF \\
\hline 17-NMe ${ }_{2}$ & 100:0 & 21360 & 91. & 0 & 100 & 91 & -78 & THF \\
\hline 17-NMe & $0: 100$ & 25200 & 0 & 76 & 87 & 87 & -78 to 0 & THF \\
\hline $17-\mathrm{NMe}_{2}$ & $90: 10$ & 1800 & 46 & 5 & 58 & 88 & -42 & THF \\
\hline 17-NMe & $76: 24$ & 7200 & 34 & 11 & 67 & 67 & -42 & THF \\
\hline 17-NMe & $37: 63$ & 64800 & 12.5 & 21 & 49 & 68 & -42 & THF \\
\hline 17-NMe & 7:93 & 147600 & 3 & 41 & 50 & 88 & -42 & THF \\
\hline 17-H & 100:0 & 20 & 100 & 0 & 100 & 100 & -78 & THF \\
\hline 17-H & $95: 5$ & 30 & 75 & 4 & 93 & 85 & -78 & THF \\
\hline 17-H & $83: 17$ & 300 & 64 & 13 & 86 & 90 & -78 & THF \\
\hline 17-H & $84: 16$ & 600 & 69 & 13.5 & 100 & 82.5 & -78 & THF \\
\hline 17-H & $64: 36$ & 1800 & 56 & 31 & 89 & 98 & -78 & THF \\
\hline 17-H & $55: 45$ & 9030 & 43.4 & 36.1 & 100 & 79.5 & -78 & $\mathrm{THF}$ \\
\hline 17-H & $98: 2$ & 12 & 78 & 2 & 100 & 80 & -78 & $\mathrm{Et}_{2} \mathrm{O}$ \\
\hline
\end{tabular}


2-Phenyl-3-hydroxy-2-methoxy-3,6-dimethylhex-4-enenitrile (20-H). ${ }^{1} \mathrm{H}$ NMR (300 MHz, $\left.\mathrm{CDCl}_{3}\right)$, $(62: 38 \mathrm{dr}$ after $20 \mathrm{sec}$, major diastereomer marked with *): $\delta$ 7.37-6.71 $(\mathrm{m}, 5 \mathrm{H}), 5.36$ (sextet, $\mathrm{J}=1.5 \mathrm{~Hz}, 1 \mathrm{H})$, $3.42(\mathrm{~s}, 3 \mathrm{H}), 3.41(\mathrm{~s}, 3 \mathrm{H})^{*}, 1.71(\mathrm{~d}, \mathrm{~J}=1.5 \mathrm{~Hz}, 3 \mathrm{H})^{*}, 1.70(\mathrm{~d}, \mathrm{~J}=1.5 \mathrm{~Hz}, 3 \mathrm{H})^{*}, 1.67(\mathrm{~d}, \mathrm{~J}=1.5 \mathrm{~Hz}, 3 \mathrm{H}), 1.53$ $(\mathrm{d}, \mathrm{J}=1.5 \mathrm{~Hz}, 3 \mathrm{H}), 1.46(\mathrm{~s}, 3 \mathrm{H}), 1.35(\mathrm{~s}, 3 \mathrm{H}) * .{ }^{13} \mathrm{C} \mathrm{NMR}\left(75.4 \mathrm{MHz}, \mathrm{CDCl}_{3}\right): \delta 138.0(\mathrm{C}), 132.8(\mathrm{C})$, 129.13*, $129.05(\mathrm{CH}), 128.1(\mathrm{CH}), 128.03^{*}(\mathrm{CH}), 127.96^{*}(\mathrm{CH}), 127.9(\mathrm{CH}), 124.4^{*}(\mathrm{CH}), 123.8(\mathrm{CH})$, $118.2(\mathrm{CN}), 88.8^{*}(\mathrm{C}), 88.6(\mathrm{C}), 78.0(\mathrm{C}), 54.96\left(\mathrm{CH}_{3}\right), 54.98^{*}\left(\mathrm{CH}_{3}\right), 27.8\left(\mathrm{CH}_{3}\right), 24.6\left(\mathrm{CH}_{3}\right), 24.2^{*}\left(\mathrm{CH}_{3}\right)$, 18.8* $\left(\mathrm{CH}_{3}\right), 18.5\left(\mathrm{CH}_{3}\right)$. HRMS (EI) (m/z): calcd. for $\mathrm{C}_{14} \mathrm{H}_{18} \mathrm{NO}_{2}\left(\mathrm{M}-\mathrm{OH}^{+}\right), 228.1388$; found 228.1383 .

2-Phenyl-2-methoxy-3, 3-dimethyl-5-oxohexanenitrile (21-H). ${ }^{1} \mathrm{H} \mathrm{NMR}\left(250 \mathrm{MHz}, \mathrm{CDCl}_{3}\right): \delta 7.43$ (m, $5 \mathrm{H}), 3.36(\mathrm{~s}, 3 \mathrm{H}), 2.75-2.55(\mathrm{AB}$ quartet, $\mathrm{J}=16.0 \mathrm{~Hz}, 2 \mathrm{H}), 2.18(\mathrm{~s}, 3 \mathrm{H}), 1.17(\mathrm{~s}, 3 \mathrm{H}), 1.12(\mathrm{~s}, 3 \mathrm{H}),{ }^{13} \mathrm{C} \mathrm{NMR}$ $\left(75.4 \mathrm{MHz}, \mathrm{CDCl}_{3}\right): \delta 207.3(\mathrm{C}), 132.6(\mathrm{C}), 129.1(\mathrm{CH}), 128.1(\mathrm{CH}), 128.0(\mathrm{CH}), 118.1(\mathrm{CN}), 89.2(\mathrm{C}), 54.8$ $\left(\mathrm{CH}_{3}\right), 48.8\left(\mathrm{CH}_{2}\right), 42.8(\mathrm{C}), 32.7\left(\mathrm{CH}_{3}\right), 22.4\left(\mathrm{CH}_{3}\right), 20.6\left(\mathrm{CH}_{3}\right)$. HRMS (EI) (m/z): calcd. for $\mathrm{C}_{15} \mathrm{H}_{19} \mathrm{NO}_{2}$ $\left(\mathrm{M}^{+}\right), 245.1416$; found 245.1411.

2-(4-Cyanophenyl)-2-methoxy-3, 3-dimethyl-5-oxo-hexanenitrile (21-CN). ${ }^{1} \mathrm{H}$ NMR (300 MHz, $\mathrm{CDCl}_{3}$ ): $\delta$ 7.76-7.57 (AA'BB', 4H), 3.35 (s, 3H), 2.73-2.54 (AB quartet, J = 14.0 Hz, 2H), 2.17 (s, 3H), 1.15 $(\mathrm{s}, 3 \mathrm{H}), 1.09(\mathrm{~s}, 3 \mathrm{H}) .{ }^{13} \mathrm{C} \mathrm{NMR}\left(75.4 \mathrm{MHz}, \mathrm{CDCl}_{3}\right): \delta 206.4(\mathrm{C}), 138.2(\mathrm{C}), 131.8(\mathrm{CH}), 128.9(\mathrm{CH}), 117.8$ $(\mathrm{CN}), 117.1(\mathrm{CN}), 113.4(\mathrm{C}), 88.8(\mathrm{C}), 55.1\left(\mathrm{CH}_{3}\right), 48.6\left(\mathrm{CH}_{2}\right), 43.0(\mathrm{C}), 32.4\left(\mathrm{CH}_{3}\right), 22.2\left(\mathrm{CH}_{3}\right), 20.3$ $\left(\mathrm{CH}_{3}\right)$. HRMS (EI) (m/z): calcd. for $\mathrm{C}_{16} \mathrm{H}_{19} \mathrm{~N}_{2} \mathrm{O}_{2}\left(\mathrm{MH}^{+}\right)$, 271.1147; found 271.1434.

2-(4-Dimethylaminophenyl)-3-hydroxy-2-methoxy-3,5-dimethylhex-4-enenitrile (20-NMe $\left.{ }_{2}\right) .{ }^{1} \mathrm{H}$ NMR $\left(300 \mathrm{MHz}, \mathrm{CDCl}_{3}\right):(75: 25 \mathrm{dr}$ after $30 \mathrm{sec}$, major diastereomer marked with *) $\delta$ 7.37-6.71 (AA'BB', 4H)*, $5.36(\mathrm{bs}, 1 \mathrm{H})^{*}, 3.40(\mathrm{~s}, 3 \mathrm{H})^{*}, 3.00(\mathrm{~s}, 6 \mathrm{H})^{*}, 2.35(\mathrm{bs}, 1 \mathrm{H})^{*}, 1.78(\mathrm{~d}, \mathrm{~J}=1.5 \mathrm{~Hz}, 3 \mathrm{H})^{*}, 1.73(\mathrm{~d}, \mathrm{~J}=1.5 \mathrm{~Hz}$, $3 \mathrm{H}), 1.35(\mathrm{~s}, 3 \mathrm{H}) * .{ }^{13} \mathrm{C} \mathrm{NMR}\left(75.4 \mathrm{MHz}, \mathrm{CDCl}_{3}\right): \delta 150.8(\mathrm{C}), 137.5(\mathrm{C}), 128.9(\mathrm{CH}), 124.9(\mathrm{CH}), 119.5$ (C), $111.3(\mathrm{CH}), 88.7(\mathrm{C}), 78.1(\mathrm{C}), 54.5\left(\mathrm{CH}_{3}\right), 40.2\left(\mathrm{CH}_{3}\right), 27.8\left(\mathrm{CH}_{3}\right), 24.0\left(\mathrm{CH}_{3}\right), 18.8\left(\mathrm{CH}_{3}\right)$. HRMS (ESI) (m/z): calcd. for $\mathrm{C}_{17} \mathrm{H}_{24} \mathrm{O}_{2} \mathrm{~N}_{2} \mathrm{Na}\left(\mathrm{MNa}^{+}\right), 311.1735$; found 311.1738 .

2-(4-Dimethylaminophenyl)-2-methoxy-3, 3-dimethyl-5-oxo-hexanenitrile (21-NMe $)$. ${ }^{1} \mathrm{H}$ NMR (300 $\mathrm{MHz}, \mathrm{CDCl}_{3}$ ): $\delta$ 7.34-6.38 (AA'BB', 4H), 3.32 (s, 3H), 2.98 (s, 6H), 2.73-2.52 (AB quartet, J = 15.0 Hz, 2H), $2.15(\mathrm{~s}, 3 \mathrm{H}), 1.14(\mathrm{~s}, 3 \mathrm{H}), 1.09(\mathrm{~s}, 3 \mathrm{H}) .{ }^{13} \mathrm{C} \mathrm{NMR}\left(75.4 \mathrm{MHz}, \mathrm{CDCl}_{3}\right): \delta 207.7(\mathrm{C}), 150.6(\mathrm{C}), 129.0(\mathrm{CH})$, $119.4(\mathrm{C}), 118.5(\mathrm{CN}), 113.4(\mathrm{C}), 89.1(\mathrm{C}), 54.5\left(\mathrm{CH}_{3}\right), 49.1\left(\mathrm{CH}_{2}\right), 43.0(\mathrm{C}), 40.2\left(\mathrm{CH}_{3}\right), 32.7\left(\mathrm{CH}_{3}\right), 22.5$ $\left(\mathrm{CH}_{3}\right), 20.7\left(\mathrm{CH}_{3}\right)$. HRMS (ESI) (m/z): calcd. for $\mathrm{C}_{17} \mathrm{H}_{24} \mathrm{O}_{2} \mathrm{~N}_{2} \mathrm{Na}\left(\mathrm{MNa}^{+}\right), 311.1735$; found 311.1721 .

2-(4-Chlorophenyl)-3-hydroxy-2-methoxy-3,5-dimethylhex-4-enenitrile (20-Cl). ${ }^{1} \mathrm{H}$ NMR (300 MHz, $\left.\mathrm{CDCl}_{3}\right),(70: 30 \mathrm{dr}$ after $5 \mathrm{sec}$, major diastereomer marked with *): $\delta$ 7.38-7.43 (AA'BB', 4H), 5.29 (bs, 1H), $3.42(\mathrm{~s}, 3 \mathrm{H}),(\mathrm{s}, 3.40)^{*} 1.72(\mathrm{~d}, \mathrm{~J}=1.5 \mathrm{~Hz}, 3 \mathrm{H})^{*}, 1.70(\mathrm{~d}, \mathrm{~J}=1.5 \mathrm{~Hz}, 3 \mathrm{H})^{*}, 1.68(\mathrm{~d}, \mathrm{~J}=1.5 \mathrm{~Hz}, 3 \mathrm{H}), 1.54(\mathrm{~d}, \mathrm{~J}=$ $1.5 \mathrm{~Hz}, 3 \mathrm{H}), 1.47(\mathrm{~s}, 3 \mathrm{H}) 1.36(\mathrm{~s}, 3 \mathrm{H}) * .{ }^{13} \mathrm{C} \mathrm{NMR}\left(75.4 \mathrm{MHz}, \mathrm{CDCl}_{3}\right): \delta 138.54 *(\mathrm{C}), 138.47(\mathrm{C}), 135.4(\mathrm{C})$, $131.6(\mathrm{C}), 131.5(\mathrm{C}), 129.6(\mathrm{CH}), 129.4 *(\mathrm{CH}), 128.4 *(\mathrm{CH}), 128.3(\mathrm{CH}), 124.2^{*}(\mathrm{CH}), 123.7(\mathrm{CH}), 117.9$ $(\mathrm{CN}), 88.4^{*}(\mathrm{C}), 87.9(\mathrm{C}), 78.1(\mathrm{C}), 78.0 *(\mathrm{C})$ 55.0 $\left(\mathrm{CH}_{3}\right), 54.9\left(\mathrm{CH}_{3}\right), 27.8^{*}\left(\mathrm{CH}_{3}\right), 27.7\left(\mathrm{CH}_{3}\right), 24.7\left(\mathrm{CH}_{3}\right)$, $24.4 *\left(\mathrm{CH}_{3}\right), 18.8^{*}\left(\mathrm{CH}_{3}\right), 18.6\left(\mathrm{CH}_{3}\right)$. HRMS (EI) $\left[\mathrm{MNa}^{+}\right](\mathrm{m} / \mathrm{z})$ : calcd.for $\mathrm{C}_{15} \mathrm{H}_{18} \mathrm{ClO}_{2} \mathrm{NNa}\left(\mathrm{MNa}^{+}\right)$, 302.0924; found 302.1110 .

2-(4-Chlorophenyl)-2-methoxy-3, 3-dimethyl-5-oxohexanenitrile (21-Cl). $\left.{ }^{1} \mathrm{H} \mathrm{NMR} \mathrm{(300} \mathrm{MHz}, \mathrm{CDCl}_{3}\right)$ :

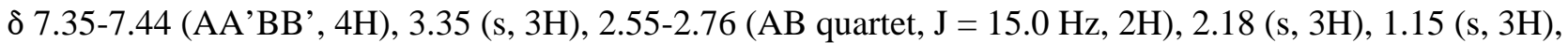
$1.10(\mathrm{~s}, 3 \mathrm{H}) .{ }^{13} \mathrm{C} \mathrm{NMR}\left(75.4 \mathrm{MHz}, \mathrm{CDCl}_{3}\right): \delta 207.0(\mathrm{C}), 135.4(\mathrm{C}), 129.5(\mathrm{CH}), 128.4(\mathrm{CH}), 117.7(\mathrm{CN}), 88.8$ (C), $54.9\left(\mathrm{CH}_{3}\right), 48.8\left(\mathrm{CH}_{2}\right), 42.9(\mathrm{C}), 32.7\left(\mathrm{CH}_{3}\right), 22.4\left(\mathrm{CH}_{3}\right), 20.5\left(\mathrm{CH}_{3}\right)$. HRMS (EI) (m/z): calcd. for $\mathrm{C}_{12} \mathrm{H}_{13} \mathrm{ClON}\left(\mathrm{M}-\mathrm{C}_{3} \mathrm{H}_{5} \mathrm{O}^{+}\right), 222.0686$; found 222.0683 .

2-(4-Trifluoromethylphenyl)-3-hydroxy-2-methoxy-3,5-dimethylhex-4-enenitrile (20-CF $)$. We were not able to obtain pure $\mathbf{2 0 -} \mathbf{C F}_{\mathbf{3}}$, since it did not survive chromatography on silica gel. The NMR spectrum was 
obtained from a mixture of 20-CF 3 and 21-CF $\mathbf{C F}_{3}{ }^{1} \mathrm{H}$ NMR $\left(300 \mathrm{MHz}, \mathrm{CDCl}_{3}\right),(59: 41 \mathrm{dr}$ after 6 sec, major diastereomer marked with *): $\delta$ 7.70-7.66 (4H, AA'BB'), $5.31(1 \mathrm{H}, \mathrm{bs})^{*}, 3.44(3 \mathrm{H}, \mathrm{s}),(3.42, \mathrm{~s})^{*}, 2.30(1 \mathrm{H}$, $\mathrm{s})^{*}, 1.73(\mathrm{~d}, \mathrm{~J}=1.5 \mathrm{~Hz}, 3 \mathrm{H})^{*}, 1.69(\mathrm{~d}, \mathrm{~J}=1.5 \mathrm{~Hz}, 3 \mathrm{H}), 1.66(\mathrm{~d}, \mathrm{~J}=1.5 \mathrm{~Hz}, 3 \mathrm{H})^{*}, 1.50(\mathrm{~d}, \mathrm{~J}=1.5 \mathrm{~Hz}, 3 \mathrm{H}), * 1.49$ $(\mathrm{s}, 3 \mathrm{H}), 1.38(\mathrm{~s}, 3 \mathrm{H}) *{ }^{13} \mathrm{C}$ NMR $\left(75.4 \mathrm{MHz}, \mathrm{CDCl}_{3}\right): \delta 138.6(\mathrm{C}), 137.2 *(\mathrm{C}), 137.1(\mathrm{C}), 131.3 *\left(\mathrm{C},{ }^{2} \mathrm{~J}_{\mathrm{C}-\mathrm{F}}=33\right.$ $\mathrm{Hz}), 131.2\left(\mathrm{C},{ }^{2} \mathrm{~J}_{\mathrm{C}-\mathrm{F}}=33 \mathrm{~Hz}\right), 129.6(\mathrm{CH}), 129.4 *(\mathrm{CH}), 128.6(\mathrm{CH}), 124.7\left(\mathrm{CH}^{3} \mathrm{~J}_{\mathrm{C}-\mathrm{F}}=4 \mathrm{~Hz}\right), 123.9 *(\mathrm{CH})$, $123.5(\mathrm{CH}), 123.3\left(\mathrm{CF}_{3}{ }^{1} \mathrm{~J}_{\mathrm{C}-\mathrm{F}}=272 \mathrm{~Hz}\right), 117.4(\mathrm{CN}), 88.4 *(\mathrm{C}), 87.8(\mathrm{C}), 78.1(\mathrm{C}), 78.0 *(\mathrm{C}) 55.0\left(\mathrm{CH}_{3}\right)$, 54.9* $\left(\mathrm{CH}_{3}\right), 27.58 *\left(\mathrm{CH}_{3}\right), 27.55\left(\mathrm{CH}_{3}\right), 24.6\left(\mathrm{CH}_{3}\right), 24.5 *\left(\mathrm{CH}_{3}\right), 18.5 *\left(\mathrm{CH}_{3}\right), 18.3\left(\mathrm{CH}_{3}\right)$.

2-(4-Trifluoromethylphenyl)-2-methoxy-3, 3-dimethyl-5-oxohexanenitrile (21- $\left.\mathbf{C F}_{\mathbf{3}}\right) .{ }^{1} \mathrm{H}$ NMR (300 $\mathrm{MHz}, \mathrm{CDCl}_{3}$ ): $\delta$ 7.57-7.73(AA'BB', 4H), 3.37 (s, 3H), 2.72-2.58 (AB quartet, J = 15.0 Hz, 2H), 2.20 (s, 3H), $1.17(\mathrm{~s}, 3 \mathrm{H}), 1.11(\mathrm{~s}, 3 \mathrm{H}) .{ }^{13} \mathrm{C} \mathrm{NMR}\left(90.6 \mathrm{MHz}, \mathrm{CDCl}_{3}\right): \delta 206.8(\mathrm{C}), 136.9(\mathrm{C}), 131.4 \mathrm{C},\left({ }^{2} \mathrm{~J}_{\mathrm{C}-\mathrm{F}}=32 \mathrm{~Hz}\right)$, $128.6(\mathrm{CH}), 125.1\left({ }^{3} \mathrm{CF}_{3} \mathrm{~J}_{\mathrm{C}-\mathrm{F}}=4 \mathrm{~Hz}\right), 123.6\left(\mathrm{CF}_{3}{ }^{1} \mathrm{~J}_{\mathrm{C}-\mathrm{F}}=272 \mathrm{~Hz}\right), 117.5(\mathrm{CN}), 88.8(\mathrm{C}), 55.0\left(\mathrm{CH}_{3}\right), 48.6$ $\left(\mathrm{CH}_{2}\right), 42.9(\mathrm{C}), 32.6\left(\mathrm{CH}_{3}\right), 22.3\left(\mathrm{CH}_{3}\right), 20.4\left(\mathrm{CH}_{3}\right)$. HRMS (EI) (m/z): calcd. for $\mathrm{C}_{32} \mathrm{H}_{36} \mathrm{~F}_{6} \mathrm{~N}_{2} \mathrm{O}_{4} \mathrm{Na}\left(2 \mathrm{MNa}^{+}\right)$, 649.2477; found 649.2482.

Lithio-(4-methoxyphenyl)acetonitrile with mesityl oxide (4-Methoxyphenyl)acetonitrile ( $34 \mu \mathrm{L}, 0.25$ mmol)was added to a $\mathrm{N}_{2}$ purged $5 \mathrm{~mL}$ long neck RBF, $3 \mathrm{~mL}$ of dry THF was added, and the system was cooled to $-78{ }^{\circ} \mathrm{C}$ under positive nitrogen pressure. $n$-BuLi $(95 \mu \mathrm{L}$ of $2.65 \mathrm{M}, 0.25 \mathrm{mmol})$ was added, stirred for 20 min, and $100 \mu \mathrm{L}$ of $2.5 \mathrm{M}$ mesityl oxide in THF was injected. The solution was stirred for the indicated time, quenched with $200 \mu \mathrm{L}$ of $3 \mathrm{M}$ propionic acid in diethyl ether and worked up as in reaction of $\mathbf{3}$ with benzylidene acetone. Data are displayed in Table S-7.

Table S-7. Time dependence of reaction of lithio- 4-methoxyphenylacetonitrile with mesityl oxide at $-78{ }^{\circ} \mathrm{C}$ in THF.

\begin{tabular}{c|c|c|c|}
\hline time/sec & $1,2: 1,4$ & \% recovery \\
\hline 7 & $100: 0$ & 102 \\
\hline 120 & $90: 10$ & 105 \\
\hline 1140 & $52: 48$ & 103 \\
\hdashline 7200 & $7: 93$ & 105
\end{tabular}

2-(4-Methoxyphenyl)-3-hydroxy-3,5-dimethylhex-4-enenitrile. ${ }^{1} \mathrm{H}$ NMR $\left(300 \mathrm{MHz}, \mathrm{CDCl}_{3}\right):(56: 44 \mathrm{dr}$ after $7 \mathrm{sec}$, major diastereomer marked with *) $\delta$ 7.23-6.79 (AA'BB', 4H)*, $5.24(\mathrm{bs}, 1 \mathrm{H})^{*}, 3.81(\mathrm{~s}, 1 \mathrm{H}), 3.77$ $(\mathrm{s}, 1 \mathrm{H})^{*}, 3.72(\mathrm{~s}, 3 \mathrm{H}), 2.07(\mathrm{bs}, 1 \mathrm{H})^{*}, 1.70(\mathrm{~d}, \mathrm{~J}=1 \mathrm{~Hz}, 3 \mathrm{H})^{*}, 1.65(\mathrm{~d}, \mathrm{~J}=1 \mathrm{~Hz}, 3 \mathrm{H}), 1.37$ (s, 3H), 1.33 (s, $3 \mathrm{H}) * .{ }^{13} \mathrm{C} \mathrm{NMR}\left(75.4 \mathrm{MHz}, \mathrm{CDCl}_{3}\right): \delta 159.5(\mathrm{C}), 137.8^{*}, 137.6(\mathrm{C}), 130.6,130.5^{*}(\mathrm{CH}), 126.5,126.4^{*}(\mathrm{CH})$, 124.04*, $123.98(\mathrm{CH}), 120.0^{*}, 119.9(\mathrm{C}), 113.9^{*}, 113.7(\mathrm{CH}), 74.4^{*}, 74.1(\mathrm{C}), 55.1\left(\mathrm{CH}_{3}\right), 49.3^{*}, 49.0(\mathrm{CH})$, 27.3, 27.2* $\left(\mathrm{CH}_{3}\right), 26.9 *, 26.8\left(\mathrm{CH}_{3}\right), 18.7\left(\mathrm{CH}_{3}\right)$. HRMS (ESI) (m/z): calcd. for $\mathrm{C}_{15} \mathrm{H}_{19} \mathrm{O}_{2} \mathrm{~N}\left(\mathrm{M}^{+}\right), 245.1416$; found 245.1419 .

2-(4-Methoxyphenyl)-3, 3-dimethyl-5-oxo-hexanenitrile. ${ }^{1} \mathrm{H}$ NMR (300 $\left.\mathrm{MHz}, \mathrm{CDCl}_{3}\right)$ : $\delta$ 7.22-6.87 (AA'BB', 4H), 4.4 (s, 1H), 3.8 (s, 3H), 2.60-2.14 (AB quartet, J = 18.0 Hz, 2H), $2.14(\mathrm{~s}, 3 \mathrm{H}), 1.20$ (s, 3H), 1.05 $(\mathrm{s}, 3 \mathrm{H}) .{ }^{13} \mathrm{C}$ NMR $\left(75.4 \mathrm{MHz}, \mathrm{CDCl}_{3}\right): \delta 207.8(\mathrm{C}), 159.4(\mathrm{C}), 130.6(\mathrm{CH}), 124.8(\mathrm{C}), 120.5(\mathrm{C}), 113.9(\mathrm{CH})$, $55.3\left(\mathrm{CH}_{3}\right), 51.1\left(\mathrm{CH}_{2}\right), 44.7(\mathrm{CH}), 36.9(\mathrm{C}), 31.8\left(\mathrm{CH}_{3}\right), 25.1\left(\mathrm{CH}_{3}\right), 24.4\left(\mathrm{CH}_{3}\right)$. HRMS (ESI) (m/z): calcd. for $\mathrm{C}_{15} \mathrm{H}_{19} \mathrm{O}_{2} \mathrm{~N}(\mathrm{M}+), 245.1416$; found 245.1419. 


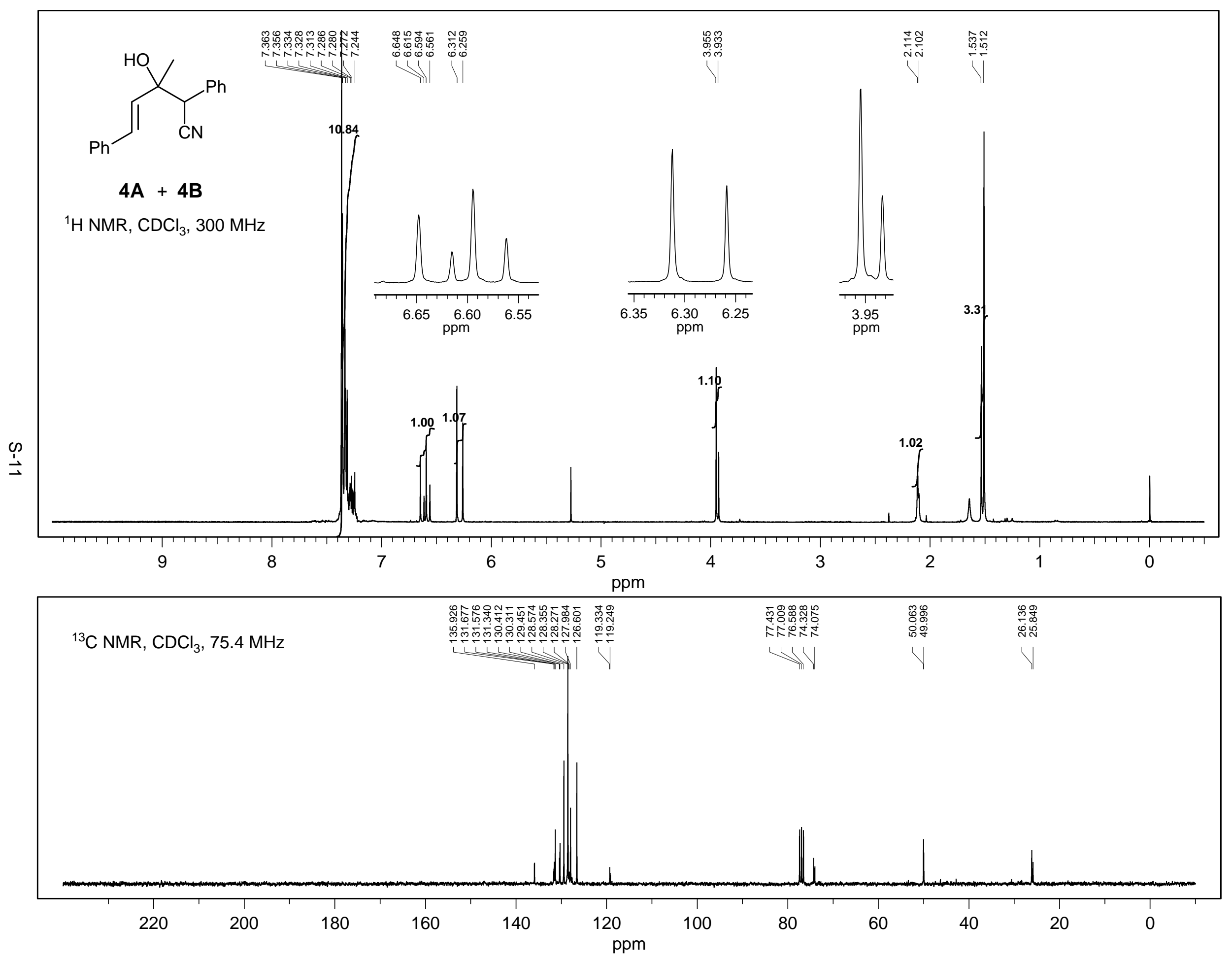




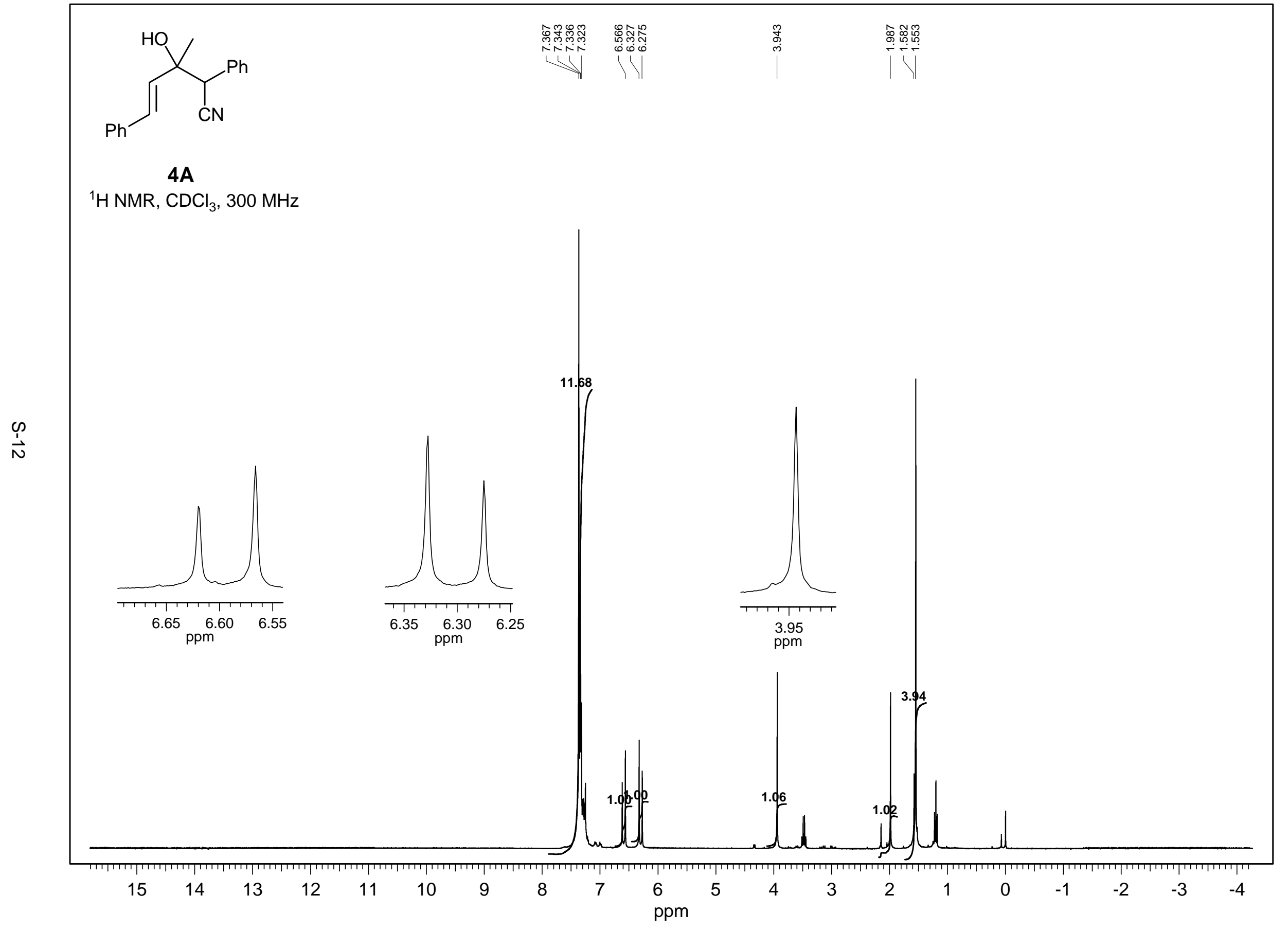




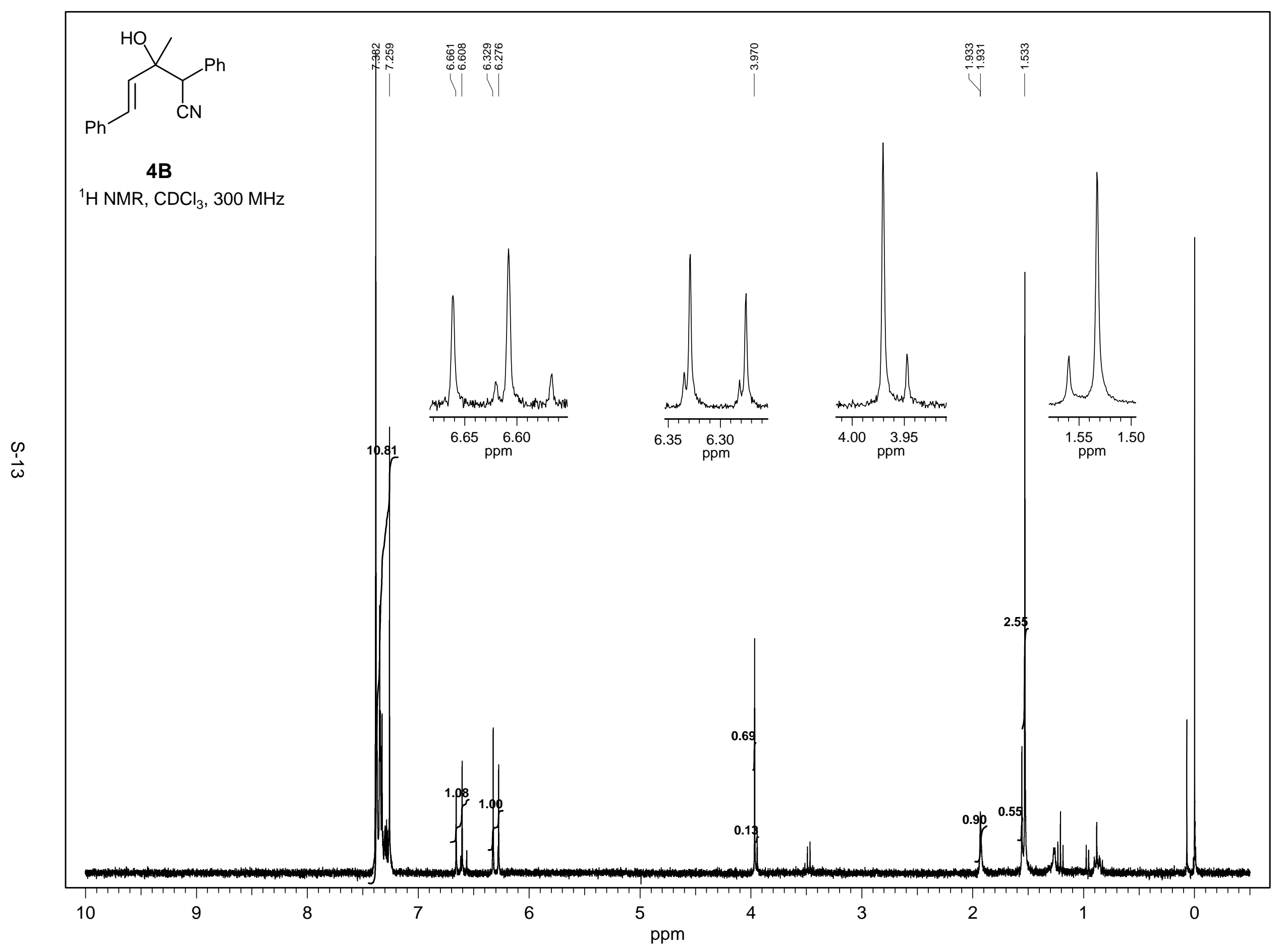




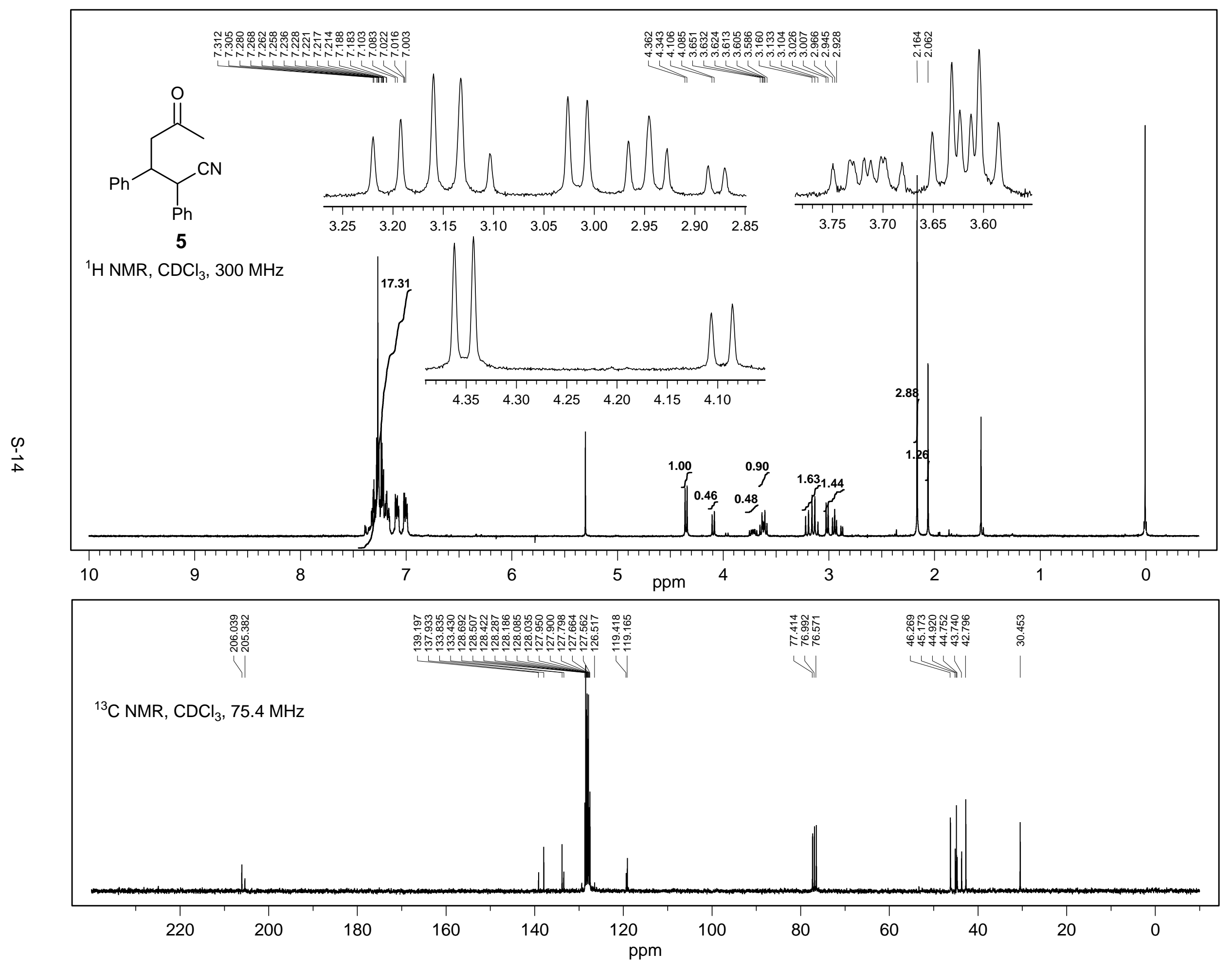




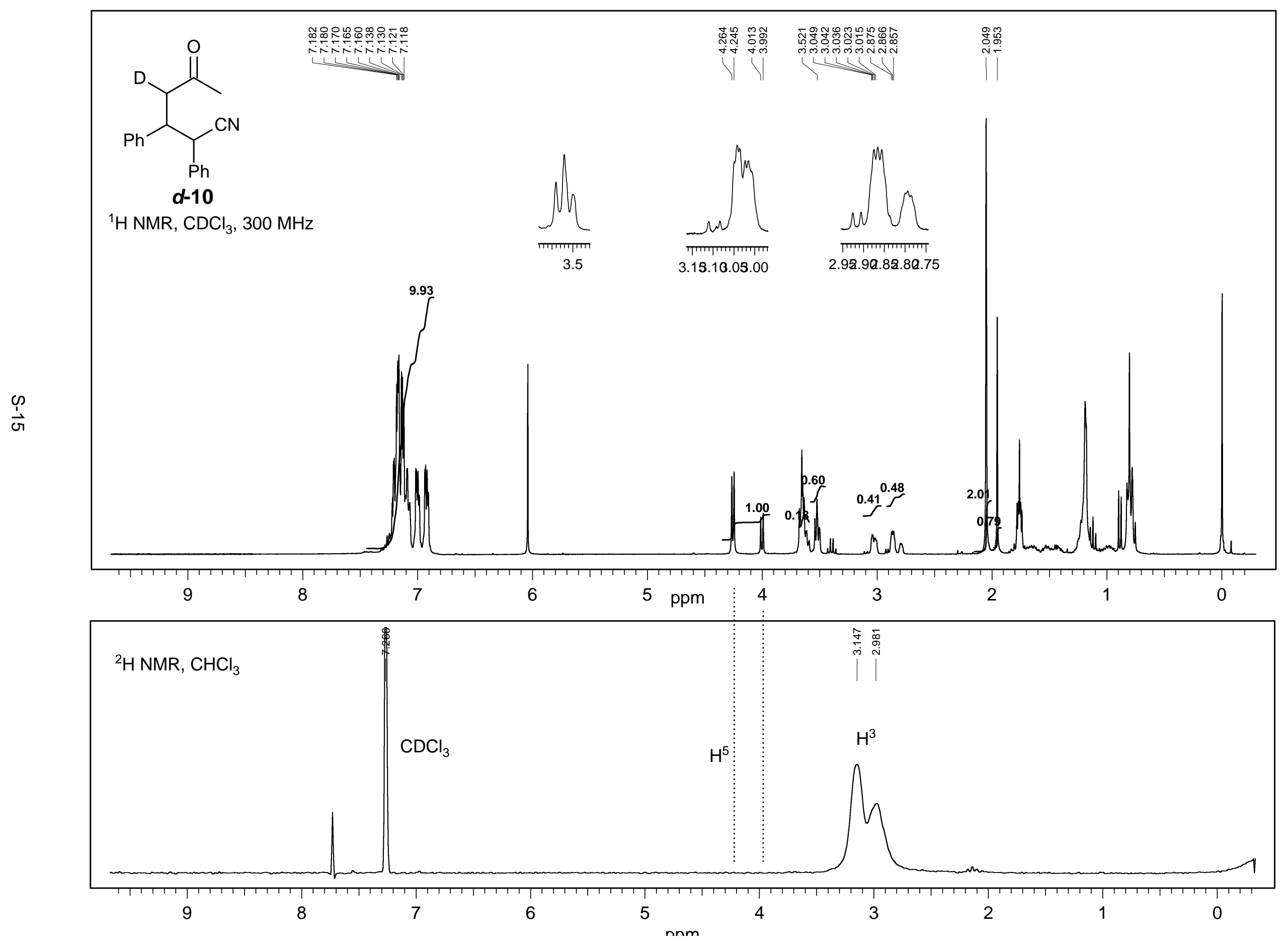



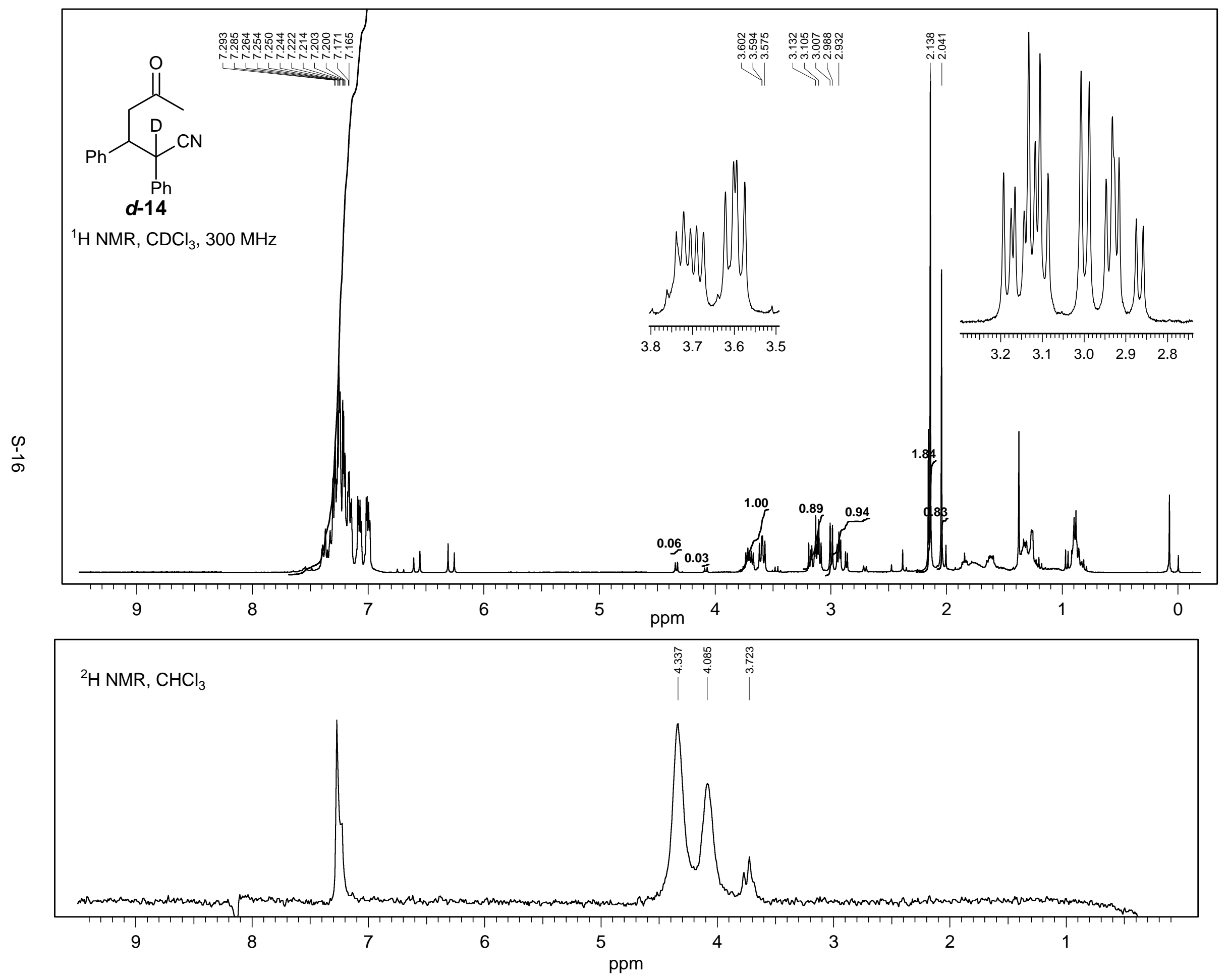


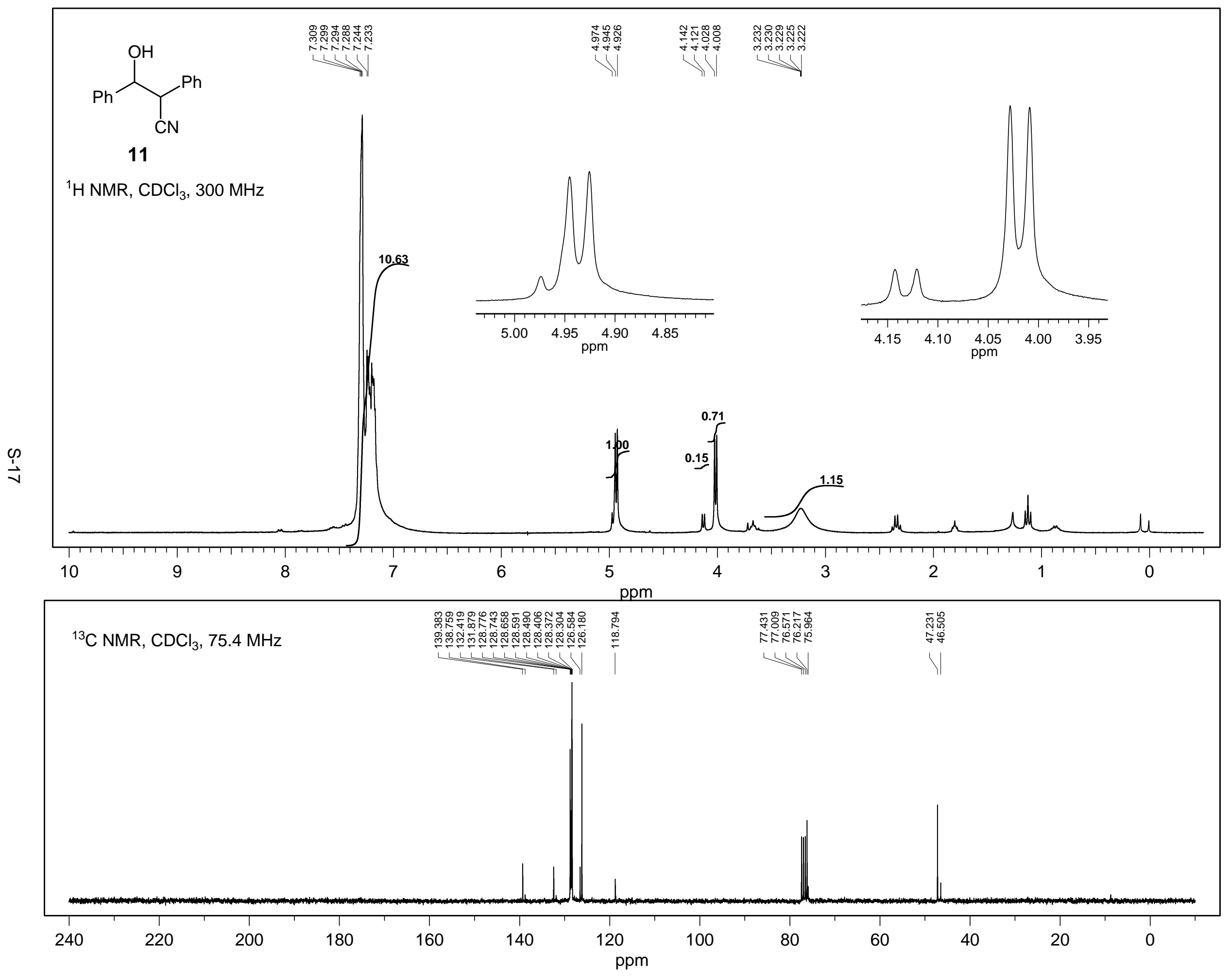




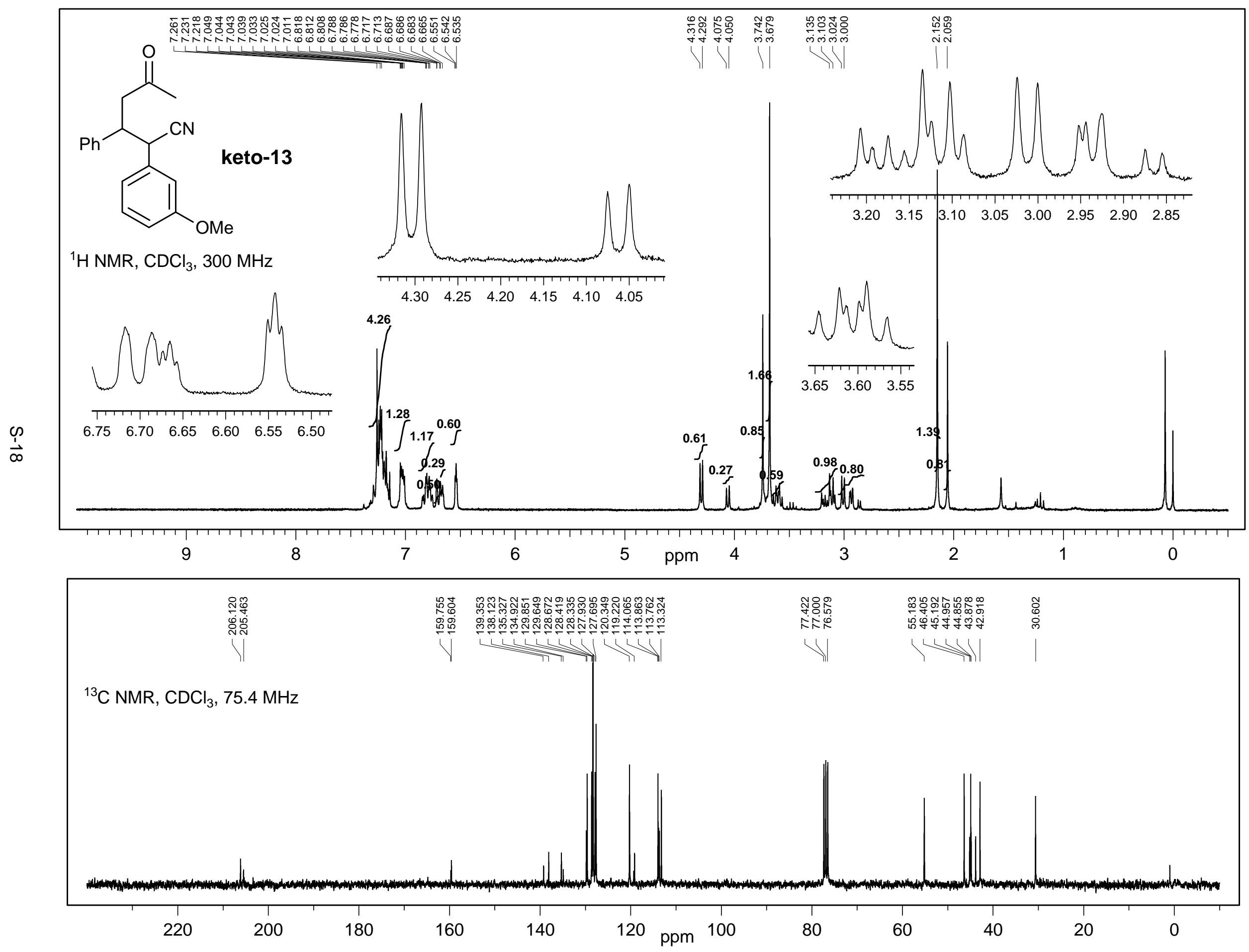




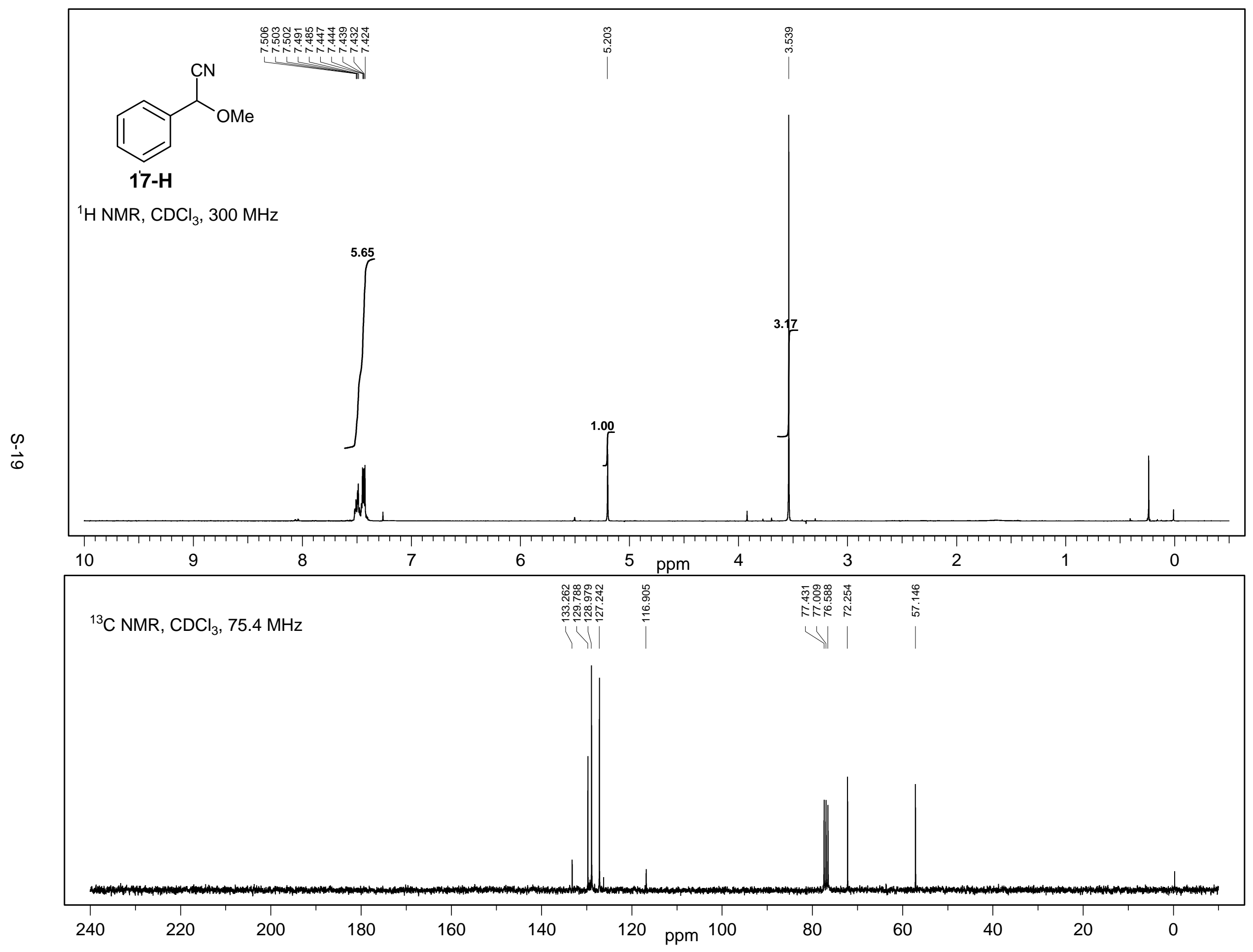




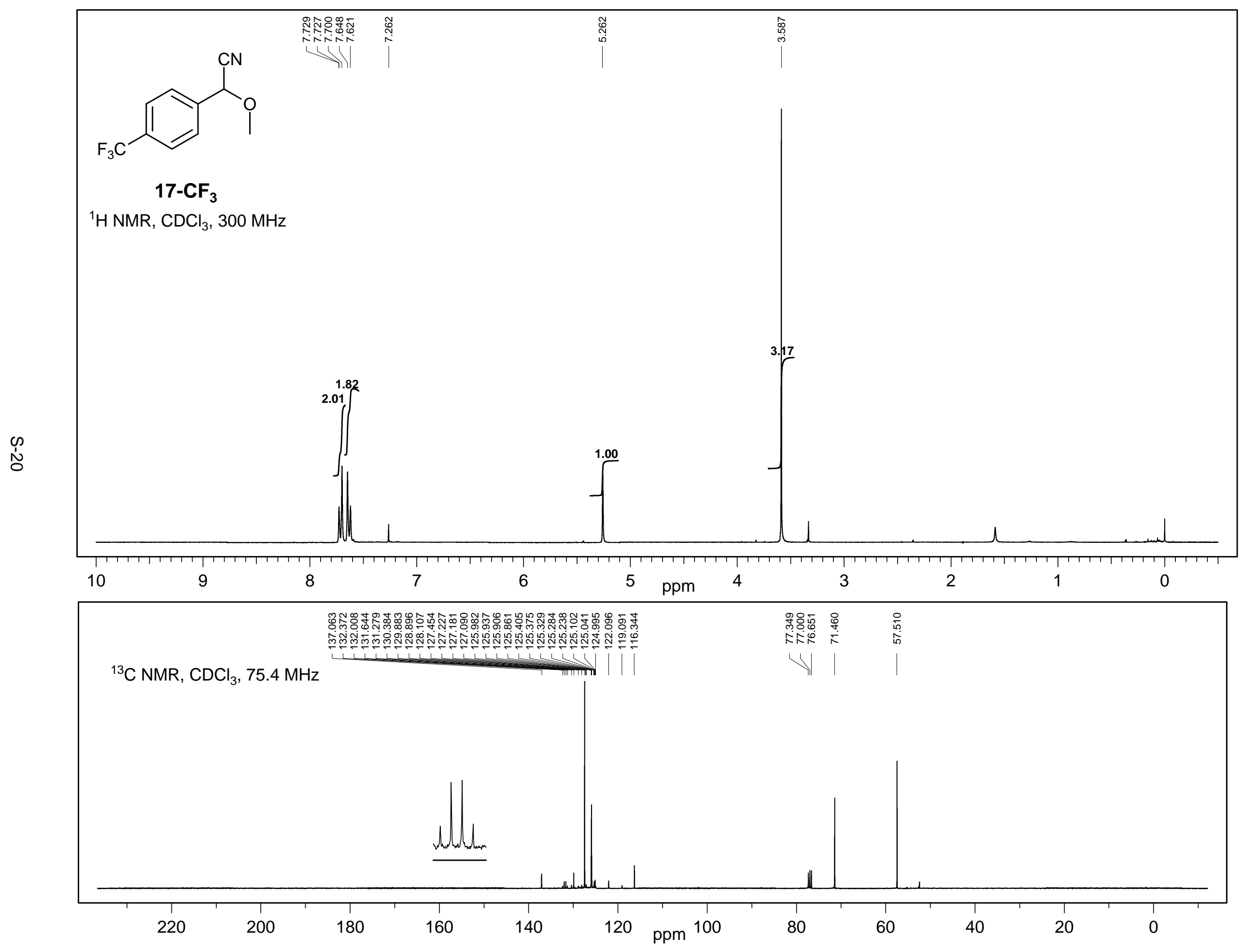




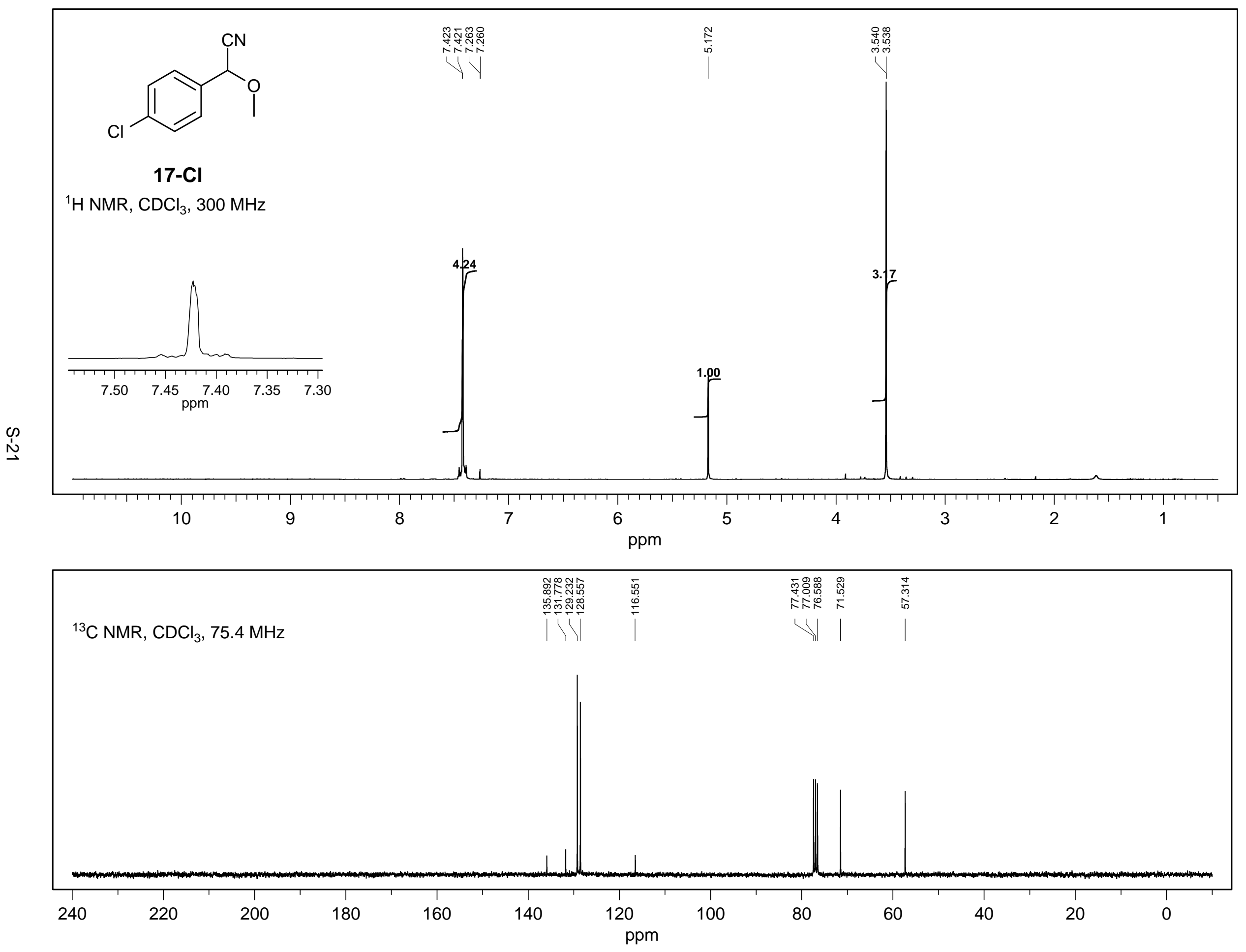




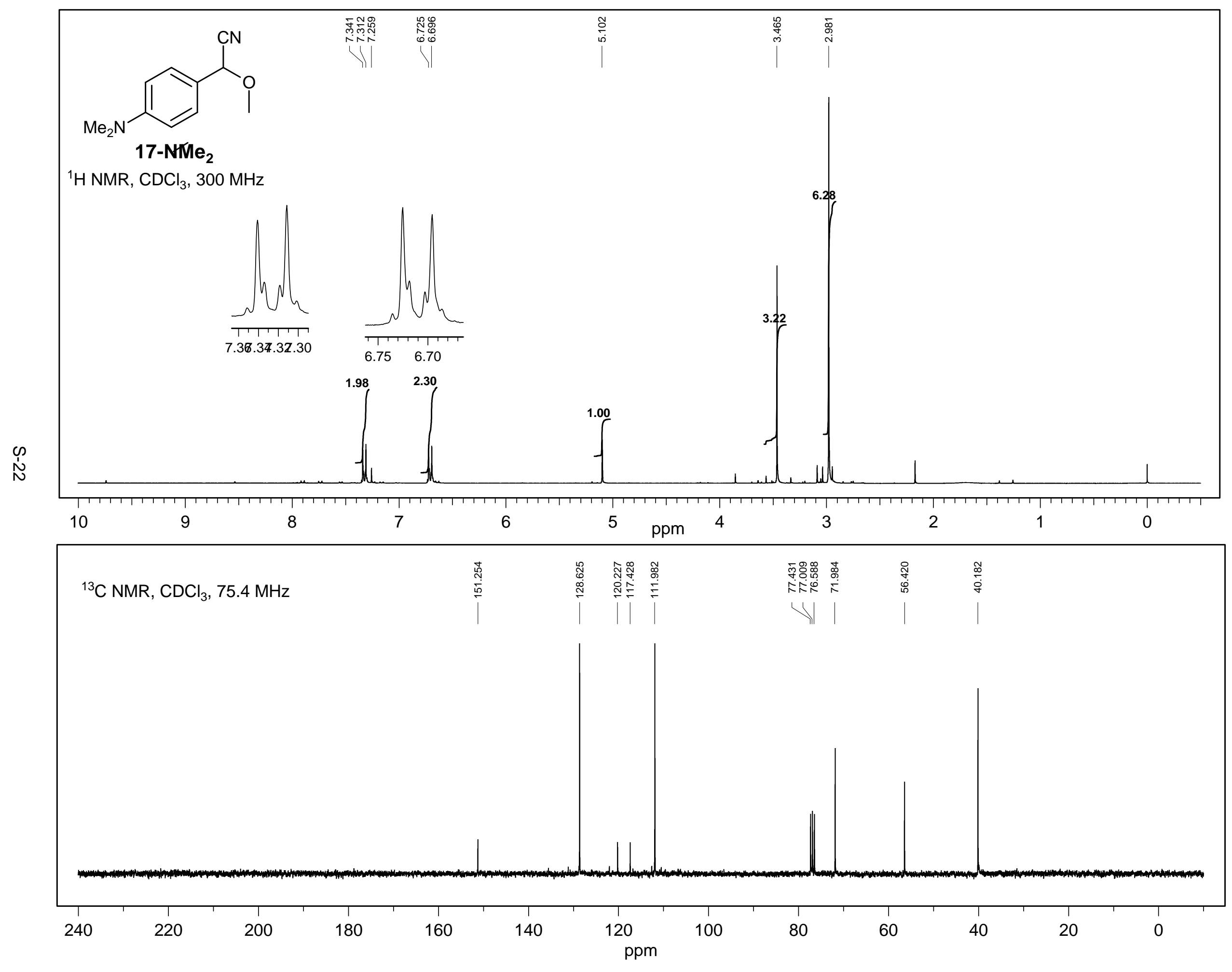



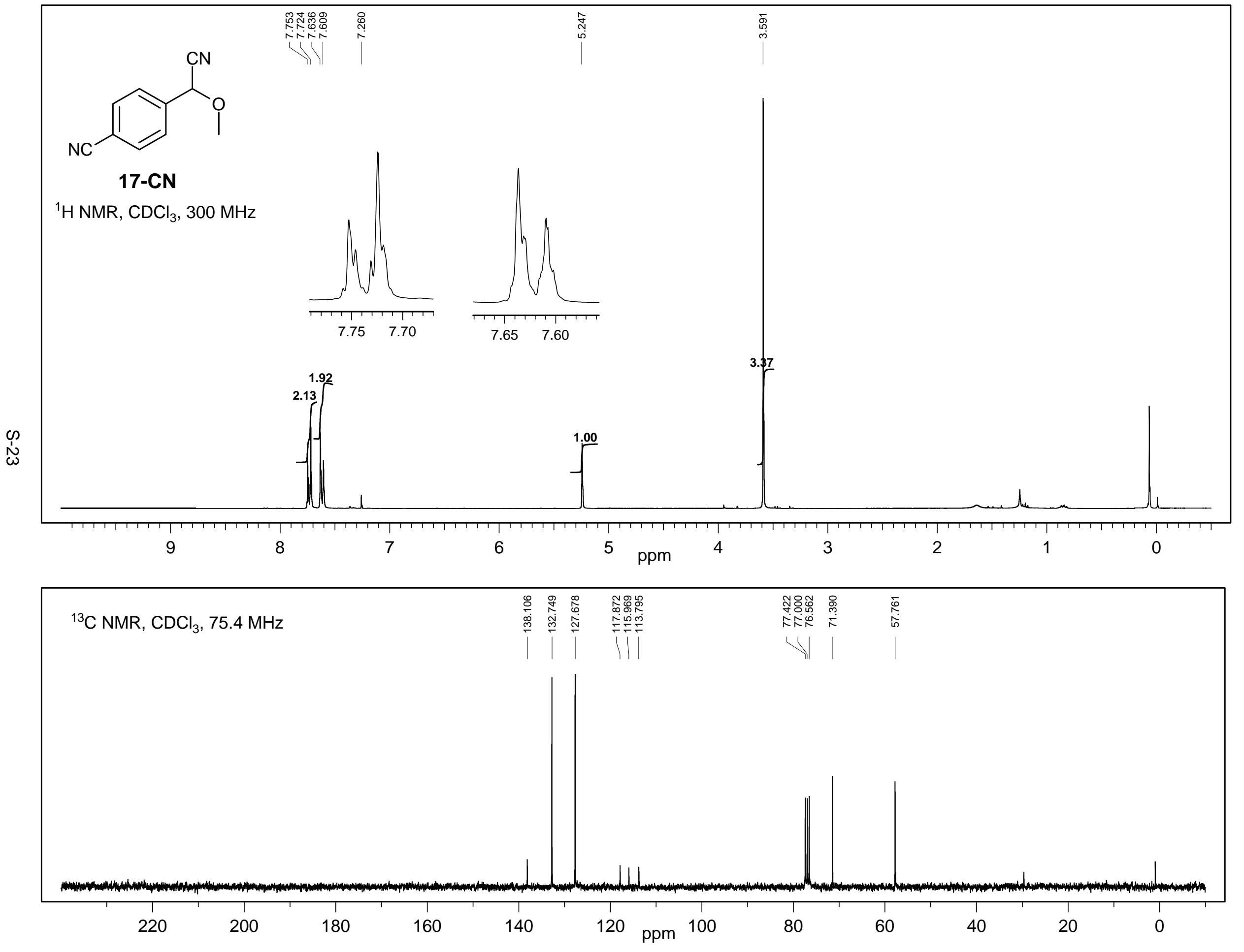


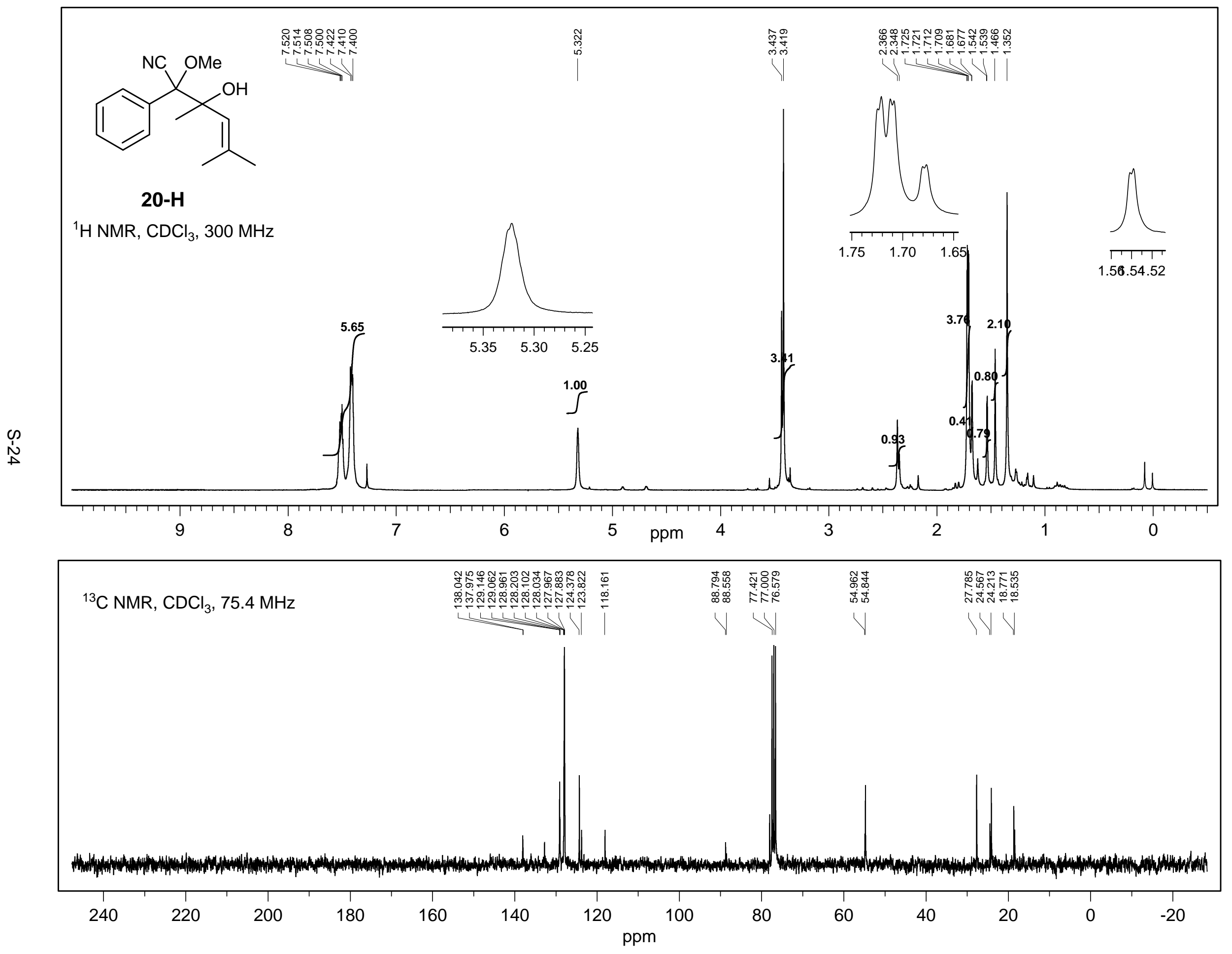




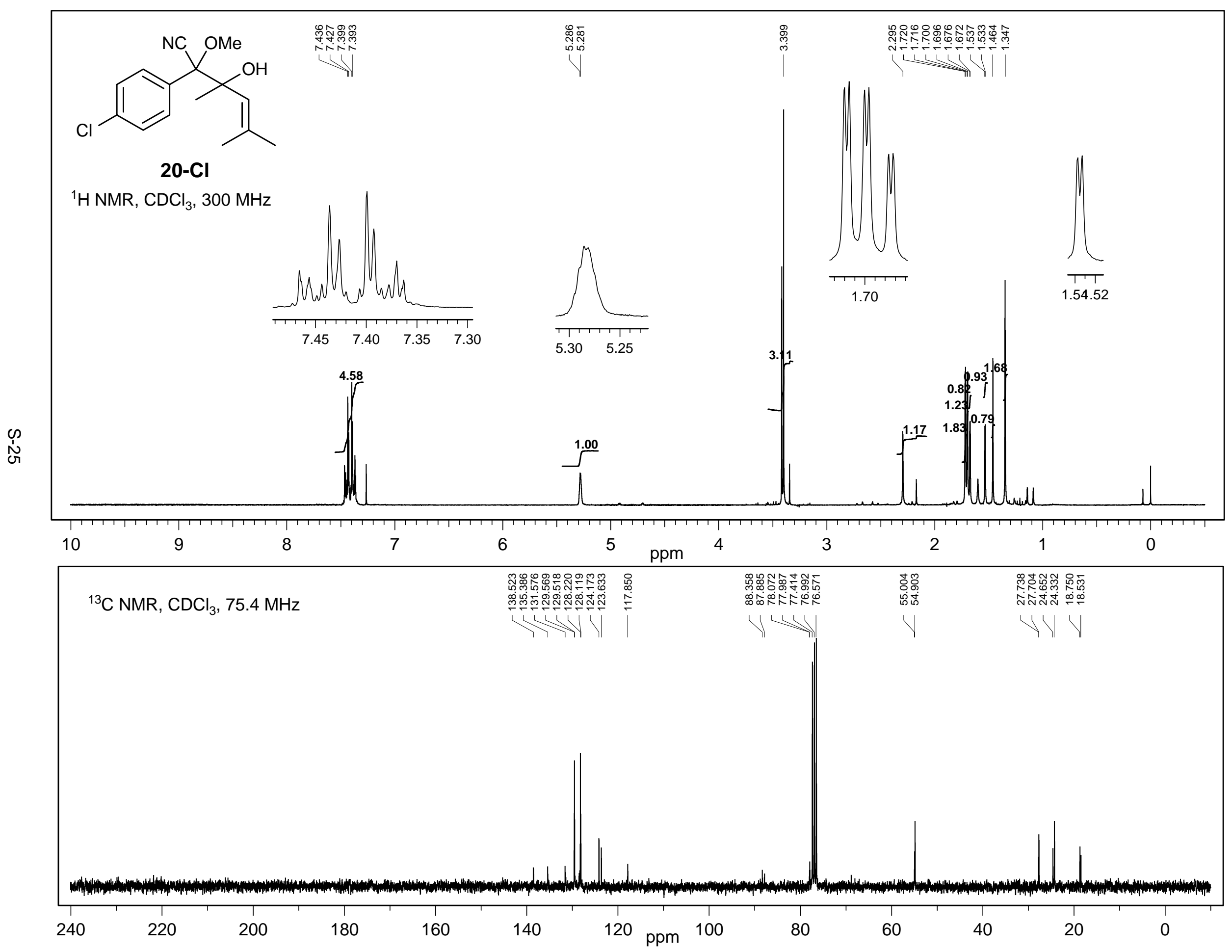




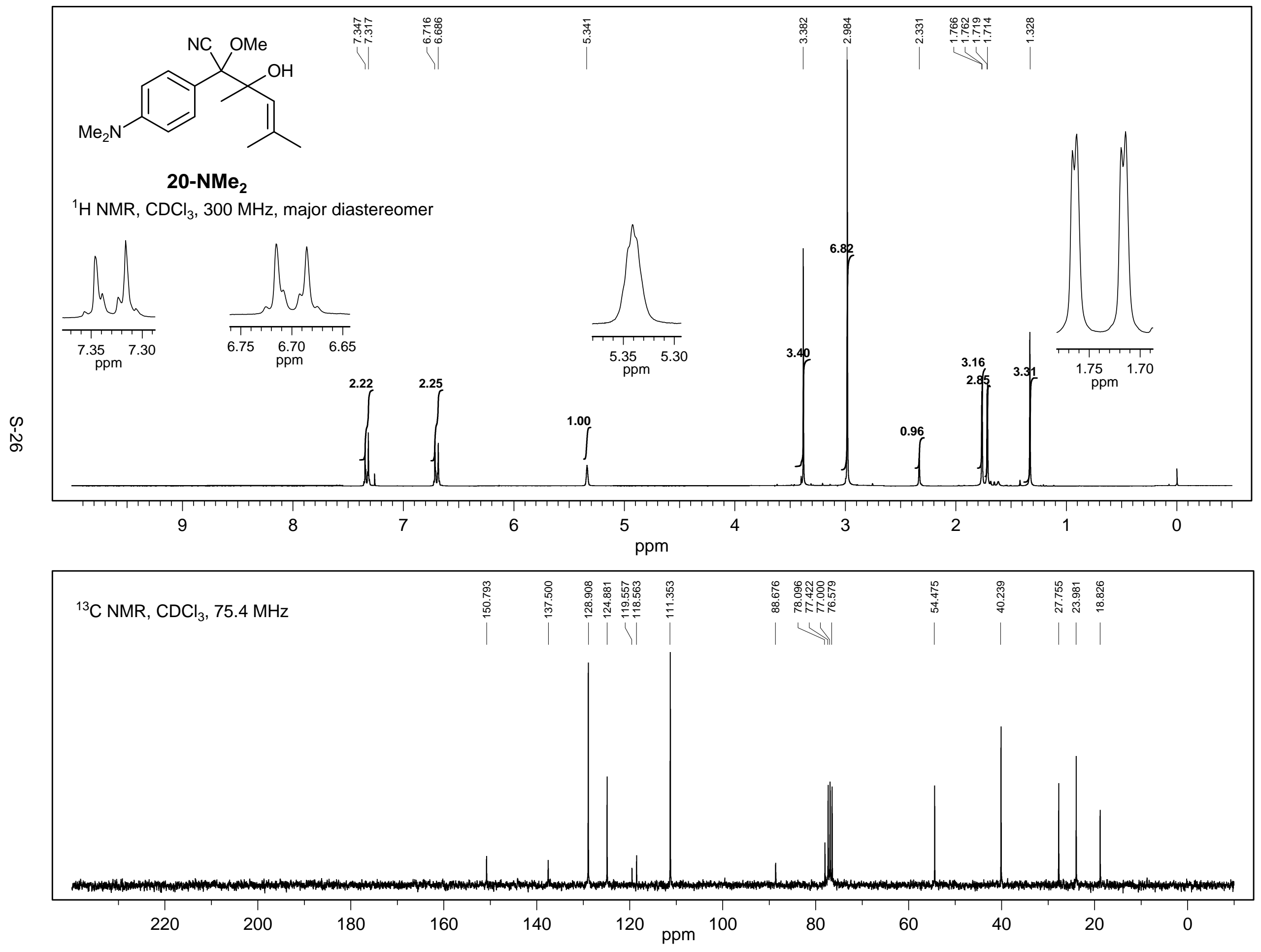




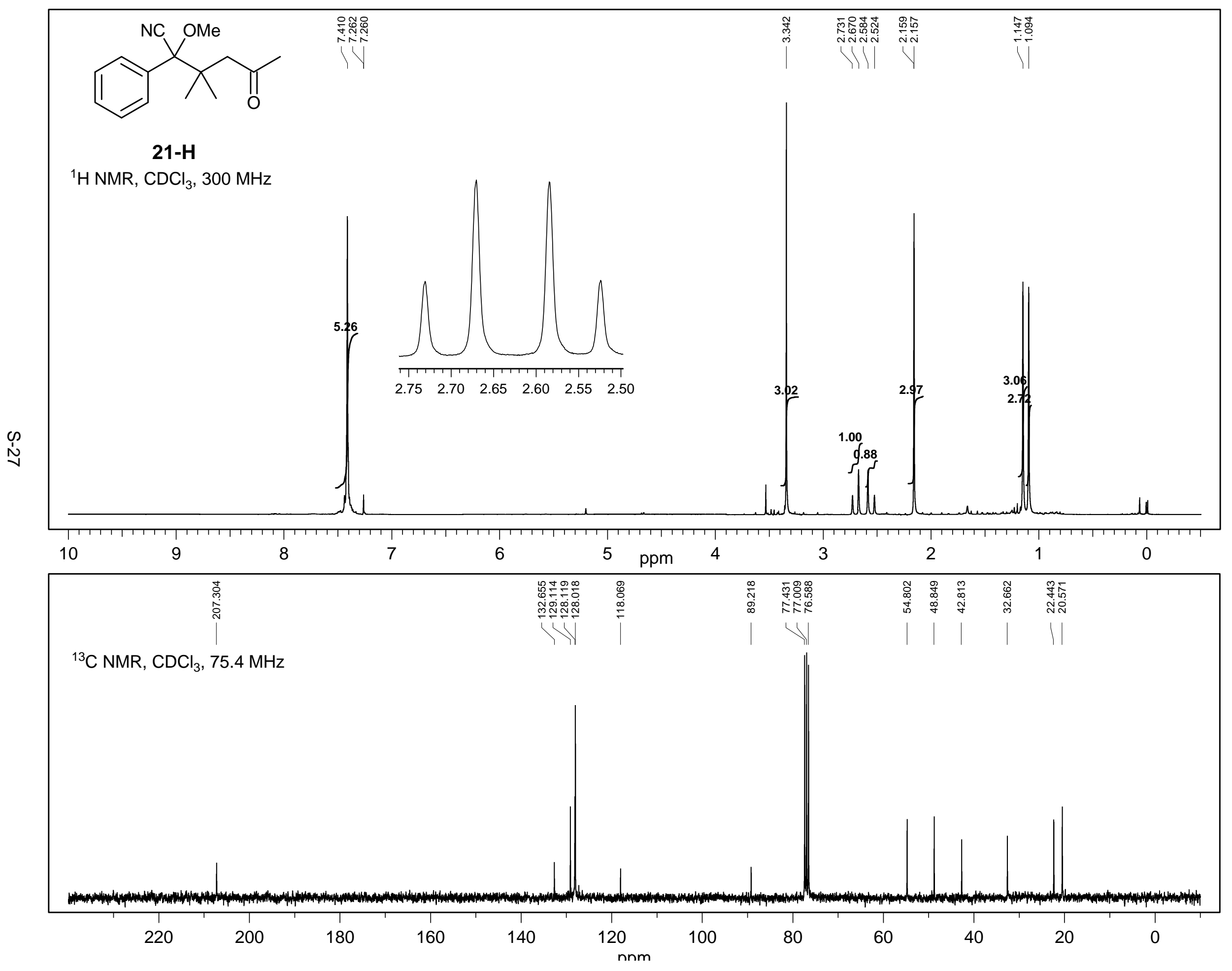




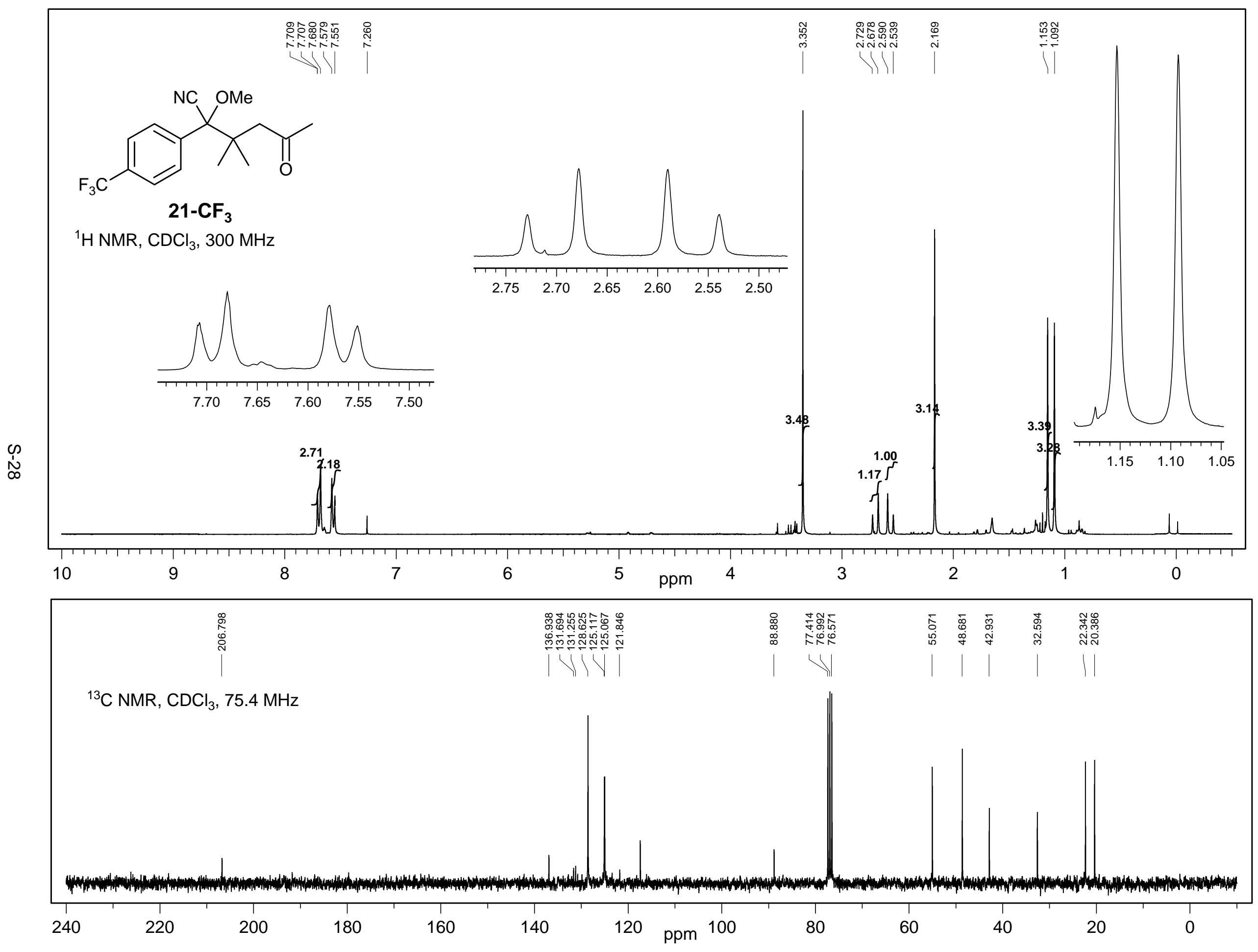




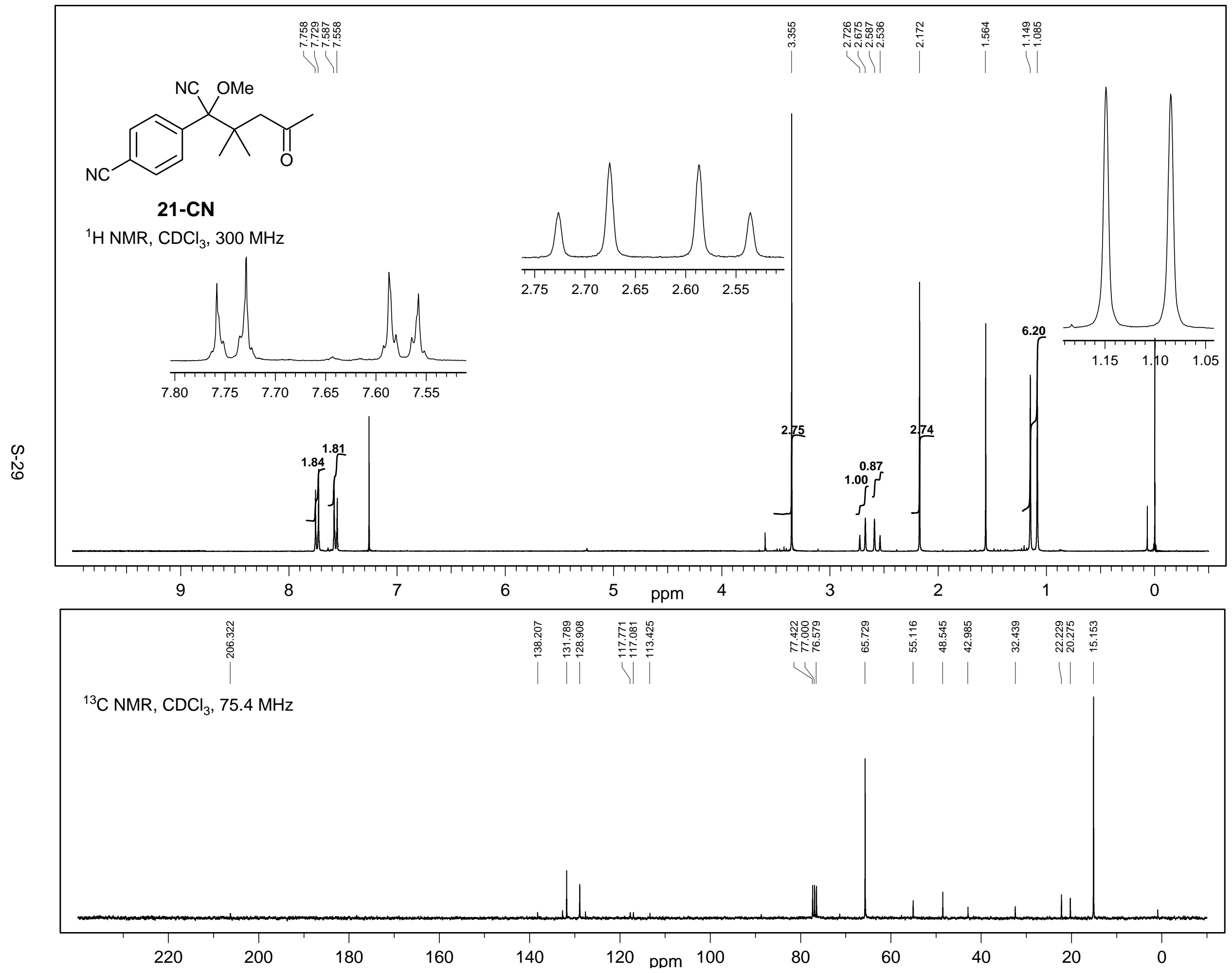




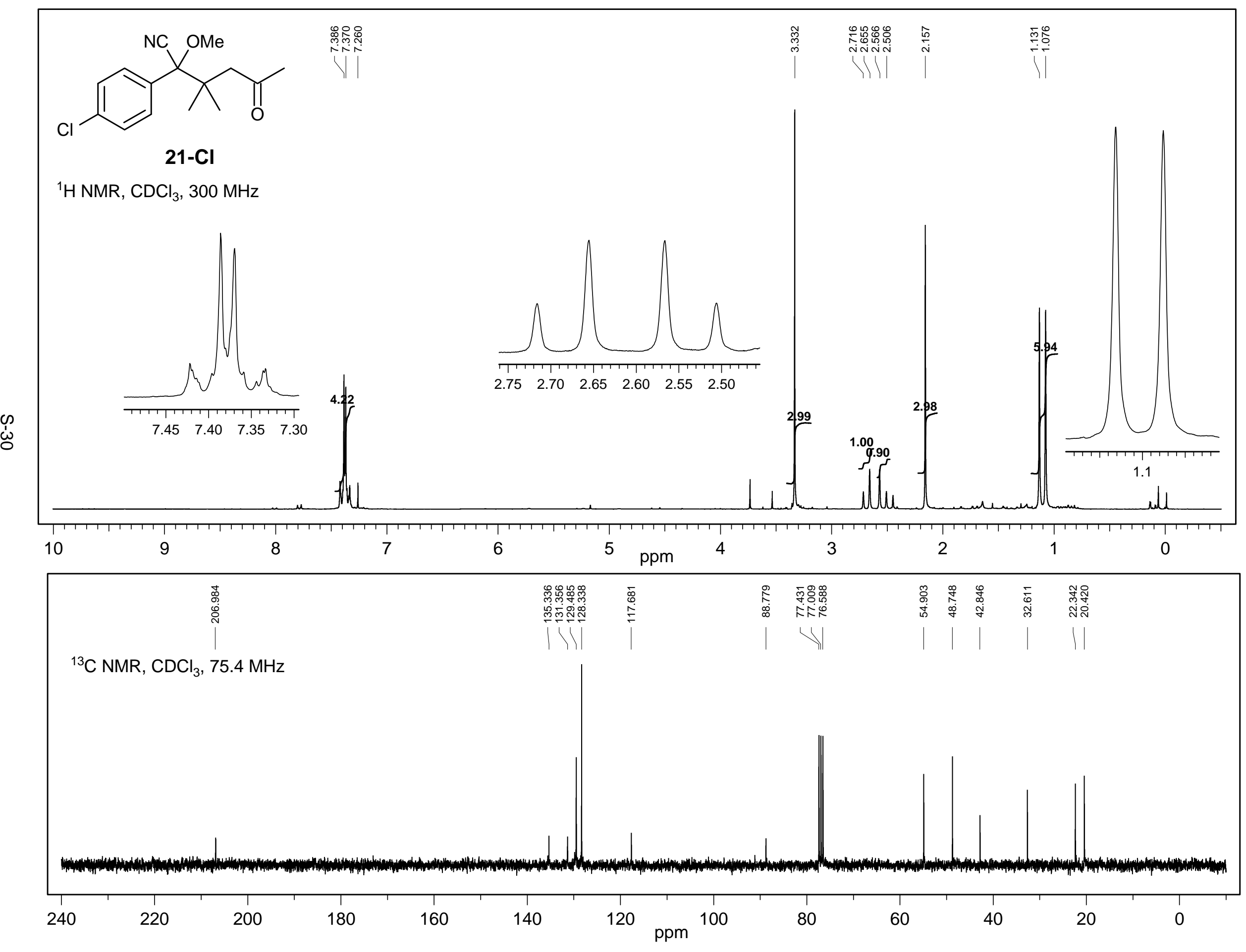




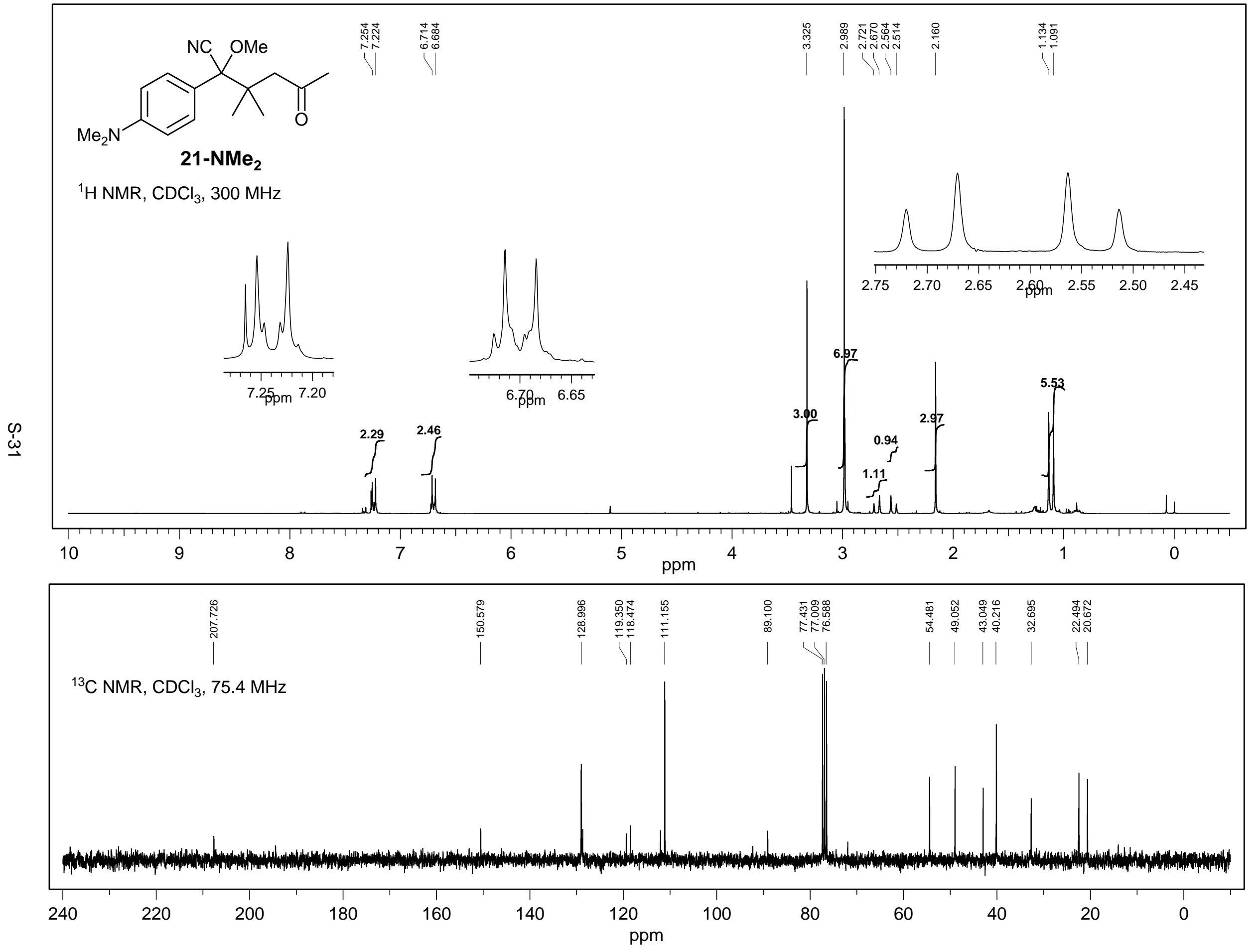




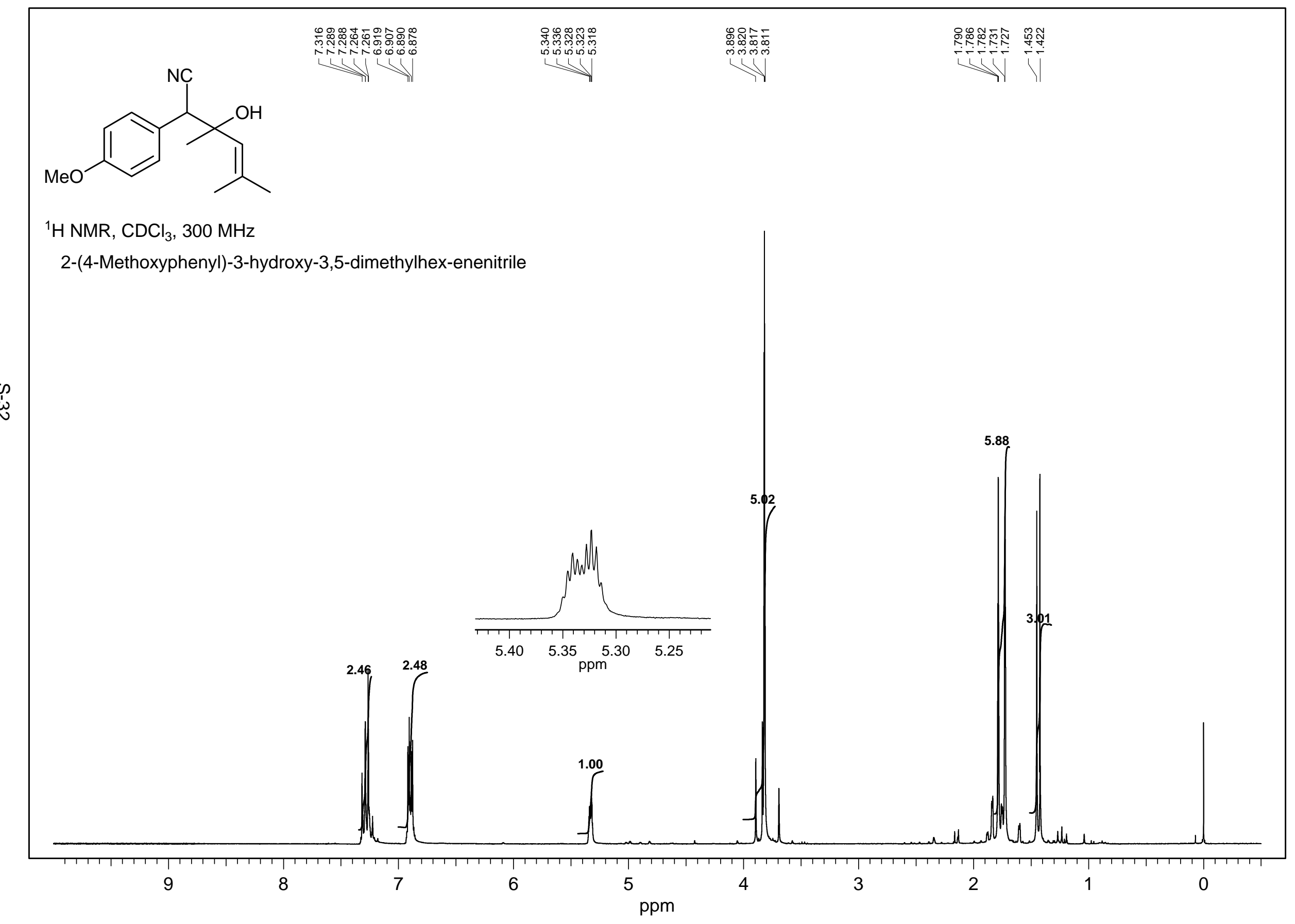




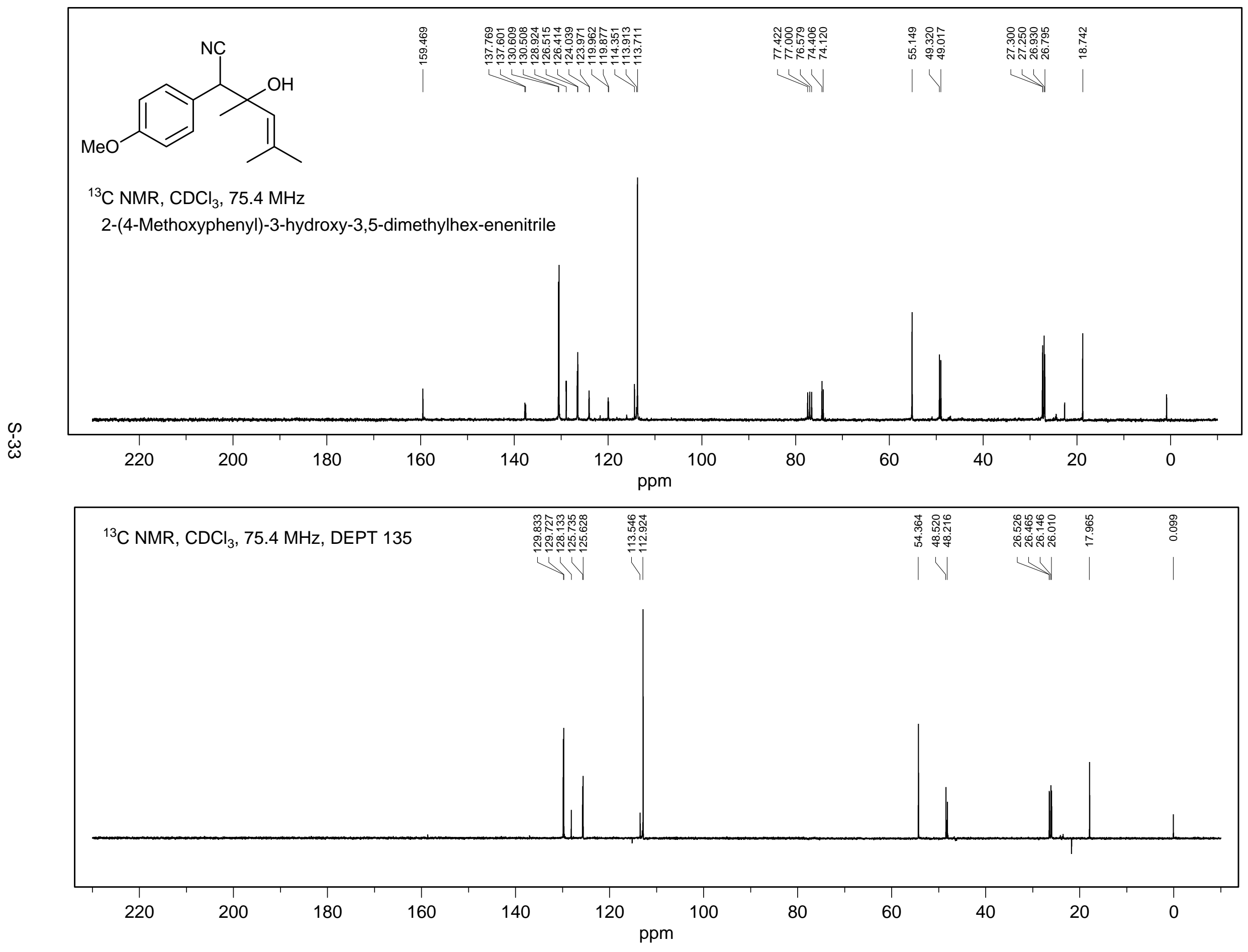




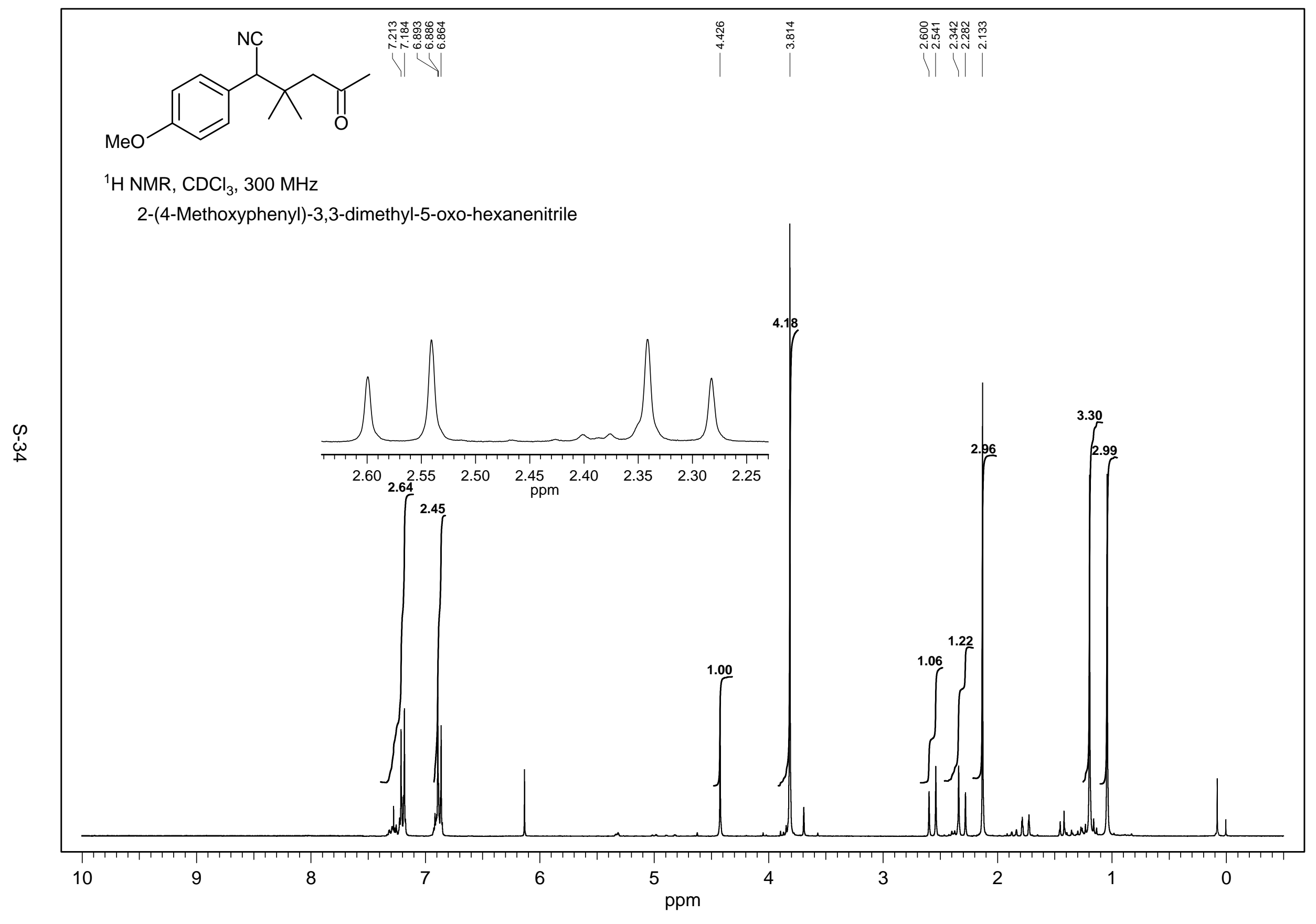




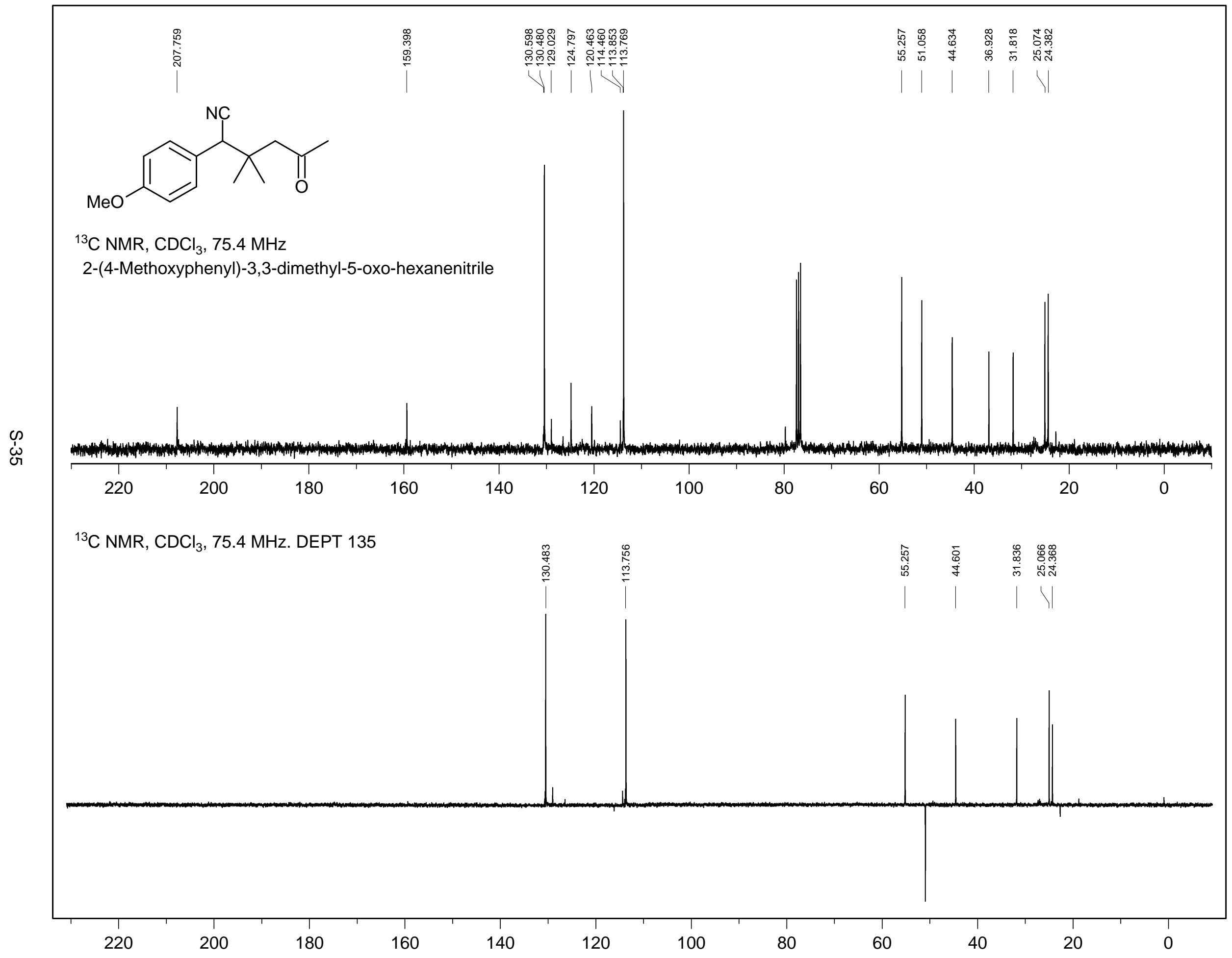

